\title{
THE NEXT GENERATION VIRGO CLUSTER SURVEY-INFRARED (NGVS-IR). I. A NEW NEAR-ULTRAVIOLET, OPTICAL, AND NEAR-INFRARED GLOBULAR CLUSTER SELECTION TOOL*
}

\author{
Roberto P. Muñoz ${ }^{1,2}$, Thomas H. Puzia ${ }^{1}$, Ariane Lançon ${ }^{2}$, Eric W. Peng ${ }^{3,4}$, Patrick Côté ${ }^{5}$, Laura Ferrarese ${ }^{5}$, \\ John P. Blakeslee ${ }^{5}$, Simona Mei ${ }^{6,7,8}$, Jean-Charles Cuillandre ${ }^{9}$, Patrick Hudelot ${ }^{10}$, Stéphane Courteau $^{11}$, \\ Pierre-Alain Duc ${ }^{12}$, Michael L. Balogh ${ }^{13}$, Alessandro Boselli ${ }^{14}$, Frédéric Bournaud ${ }^{12}$, Raymond G. Carlberg ${ }^{15}$,

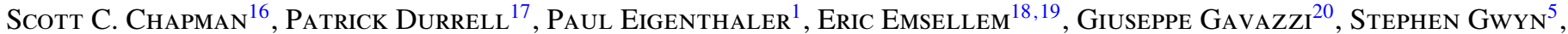

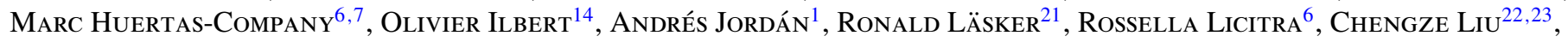 \\ Lauren MacArthur ${ }^{5}$, Alan McConnachie ${ }^{5}$, Henry Joy McCracken ${ }^{10}$, Yannick Mellier ${ }^{10}$, Chien Y. Peng ${ }^{24}$, \\ Anand Raichoor ${ }^{7}$, Matthew A. Taylor ${ }^{1}$, John L. Tonry $^{25}$, R. Brent Tully ${ }^{25}$, And HongXin Zhang ${ }^{4}$ \\ ${ }^{1}$ Instituto de Astrofísica, Facultad de Física, Pontificia Universidad Católica de Chile, \\ Av. Vicuña Mackenna 4860, 7820436 Macul, Santiago, Chile; rmunoz@astro.puc.cl \\ ${ }^{2}$ Observatoire astronomique de Strasbourg, Université de Strasbourg, CNRS, UMR 7550, 11 rue de l'Universite, F-67000 Strasbourg, France \\ ${ }^{3}$ Department of Astronomy, Peking University, Beijing 100871, China \\ ${ }^{4}$ Kavli Institute for Astronomy and Astrophysics, Peking University, Beijing 100871, China \\ ${ }^{5}$ Herzberg Institute of Astrophysics, National Research Council of Canada, Victoria, BC V9E 2E7, Canada \\ ${ }^{6}$ GEPI, Observatoire de Paris, CNRS, Université Paris Diderot, 5 Place J. Janssen, F-92190 Meudon Cedex, France \\ ${ }^{7}$ Université Paris Denis Diderot, F-75205 Paris Cedex 13, France \\ ${ }^{8}$ California Institute of Technology, Pasadena, CA 91125, USA \\ ${ }^{9}$ Canada-France-Hawaii Telescope Corporation, Kamuela, HI 96743, USA \\ ${ }^{10}$ Institut d'Astrophysique de Paris, UMR 7095 CNRS \& UPMC, 98 bis Boulevard Arago, F-75014 Paris, France \\ ${ }^{11}$ Department of Physics, Engineering Physics and Astronomy, Queen's University, Kingston, ON, Canada \\ ${ }^{12}$ Laboratoire AIM Paris-Saclay, CNRS/INSU, Université Paris Diderot, CEA/IRFU/SAp, F-91191 Gif-sur-Yvette Cedex, France \\ ${ }^{13}$ Department of Physics and Astronomy, University of Waterloo, Waterloo, ON N2L 3G1, Canada \\ ${ }^{14}$ Aix Marseille Université, CNRS, LAM (Laboratoire d'Astrophysique de Marseille) UMR 7326, F-13388 Marseille, France \\ ${ }^{15}$ Department of Astronomy and Astrophysics, University of Toronto, Toronto, ON M5S 3H4, Canada \\ ${ }^{16}$ Institute of Astronomy, University of Cambridge, Madingley Road, Cambridge CB3 OHA, UK \\ ${ }^{17}$ Department of Physics and Astronomy, Youngstown State University, One University Plaza, Youngstown, OH 44555, USA \\ ${ }^{18}$ Université de Lyon 1, CRAL, Observatoire de Lyon, 9 av. Charles André, F-69230 Saint-Genis Laval, CNRS, UMR 5574, ENS de Lyon, France \\ ${ }^{19}$ European Southern Observatory, Karl-Schwarzchild-Str. 2, D-85748 Garching, Germany \\ ${ }^{20}$ Università degli Studi di Milano-Bicocca, Piazza della Scienza 3, I-20126, Milano, Italy \\ ${ }^{21}$ Max Planck Institute for Astronomy, Königstuhl 17, D-69117 Heidelberg, Germany \\ ${ }^{22}$ Center for Astronomy and Astrophysics, Department of Physics and Astronomy, \\ Shanghai Jiao Tong University, 800 Dongchuan Road, Shangai 200240, China \\ ${ }^{23}$ INPAC, Department of Physics and Astronomy and Shanghai Key Lab for Particle Physics and Cosmology, \\ Shanghai Jiao Tong University, Shanghai 200240, China \\ ${ }^{24}$ Giant Magellan Telescope Organization, 251 South Lake Avenue, Suite 300, Pasadena, CA 91101, USA \\ ${ }^{25}$ Institute for Astronomy, University of Hawaii, 2680 Woodlawn Drive, Honolulu, HI 96822, USA \\ Received 2013 August 22; accepted 2013 October 31; published 2013 December 11
}

\begin{abstract}
The NGVS-IR project (Next Generation Virgo Cluster Survey-Infrared) is a contiguous, near-infrared imaging survey of the Virgo cluster of galaxies. It complements the optical wide-field survey of Virgo (NGVS). In its current state, NGVS-IR consists of $K_{s}$-band imaging of $4 \mathrm{deg}^{2}$ centered on M87 and $J$ - and $K_{s}$-band imaging of $\sim 16 \mathrm{deg}^{2}$ covering the region between M49 and M87. We present observations of the central $4 \mathrm{deg}^{2}$ centered on Virgo's core region. The data were acquired with WIRCam on the Canada-France-Hawaii Telescope, and the total integration time was $41 \mathrm{hr}$ distributed over 34 contiguous tiles. A survey-specific strategy was designed to account for extended galaxies while still measuring accurate sky brightness within the survey area. The average $5 \sigma$ limiting magnitude is $K_{s}=24.4 \mathrm{AB}$ mag, and the $50 \%$ completeness limit is $K_{s}=23.75 \mathrm{AB}$ mag for point-source detections, when using only images with better than 0!7 seeing (median seeing 0.54). Star clusters are marginally resolved in these image stacks, and Virgo galaxies with $\mu_{K_{s}} \simeq 24.4 \mathrm{AB}$ mag $\operatorname{arcsec}^{-2}$ are detected. Combining the $K_{s}$ data with optical and ultraviolet data, we build the $u i K_{s}$ color-color diagram, which allows a very clean color-based selection of globular clusters in Virgo. This diagnostic plot will provide reliable globular cluster candidates for spectroscopic follow-up campaigns, needed to continue the exploration of Virgo's photometric and kinematic substructures, and will help the design of future searches for globular clusters in extragalactic systems. We show that the new $u i K_{s}$ diagram displays significantly clearer substructure in the distribution of stars, globular clusters, and galaxies than the $g z K_{s}$ diagram-the NGVS + NGVS-IR equivalent of the $B z K$ diagram that is widely used in cosmological surveys. Equipped with this powerful new tool, future NGVS-IR investigations based on the $u i K_{s}$ diagram will address the mapping and analysis of extended structures and compact stellar systems in and around Virgo galaxies.
\end{abstract}

Key words: galaxies: clusters: individual (Virgo) - galaxies: distances and redshifts - galaxies: luminosity function, mass function - galaxies: photometry - galaxies: star clusters: general

Online-only material: color figures 


\section{INTRODUCTION}

The proximity of the Virgo galaxy cluster, located at a distance of 16.5 $\pm 0.2 \mathrm{Mpc}$ (Mei et al. 2007; Bohlin 2007), makes it one of the closest and most thoroughly studied laboratories of star and galaxy formation in the nearby universe. With about 2000 known galaxy members (Binggeli et al. 1985; Gavazzi et al. 2003), more than $10^{4}$ globular clusters (GCs) in M87 and M49 alone (Tamura et al. 2006a, 2006b; Peng et al. 2008), and a total GC population of $(6.48 \pm 1.44) \times 10^{4}$ for the entire region (P. Durrell et al. 2014, in preparation), it offers vast sample statistics to investigate the evolution of stellar populations and the formation and assembly histories of their host galaxies in a relatively dense cluster environment.

The first detailed census of the Virgo Cluster was published by Ames (1930) and consisted of a catalog of 2278 galaxies brighter than 18th magnitude. This survey led to the identification of several background clusters and to the discovery of a southern extension of the cluster, which are impressive results given the technical limitations existing at that time. A quarter of a century later, Reaves (1956) published the first comprehensive catalog of dwarf galaxies in the Virgo Cluster, based on the study of photographic plates taken with the Lick 20 inch $(0.5 \mathrm{~m})$ astrograph. This work showed the presence of a large number of dwarf elliptical galaxies beyond the Local Group and started a new phase of Virgo Cluster research. The most important contribution to assessing Virgo Cluster membership was published by Binggeli et al. (1985) in the form of the famous Virgo Cluster Catalog (VCC). This catalog consists of 2096 galaxies, covers an area of $140 \mathrm{deg}^{2}$, and is complete down to $B_{T} \leqslant 18 \mathrm{mag}$.

\subsection{The Next Generation Virgo Cluster Survey}

The VCC has been the reference standard of Virgo for more than a quarter of a century and is about to be superseded by the Next Generation Virgo Cluster Survey (NGVS; Ferrarese et al. 2012). NGVS is a multipassband optical survey conducted with MegaCam at the Canada-France-Hawaii Telescope (CFHT), on Mauna Kea, Hawaii, a 3.6 m telescope that benefits from superb seeing and transparency conditions.

The NGVS survey area covers 104 contiguous square degrees out to the virial radius around M87 and M49 to unprecedented photometric depths in the five optical filters ${ }^{26} u^{*}$ griz. With substantially subarcsecond seeing, a surface brightness sensitivity better than $\mu_{g} \simeq 29.0 \mathrm{mag} \operatorname{arcsec}^{-2}$, and a point-source detection limit of $g \simeq 25.9$ mag $(10 \sigma)$, this survey will extend our optical view of the galaxy luminosity function and the scaling relations of galaxies to luminosities more than 5 mag below the faintest VCC galaxies. ${ }^{27}$

The five optical passbands of NGVS combined with the superior spatial resolution of the images ( $i$-band seeing better than 0 ' $6, \simeq 48 \mathrm{pc}$ at Virgo's distance) provide a first classification of the sources but leave important areas of ambiguity.

\footnotetext{
* Based on observations obtained with WIRCam, a joint project of Canada-France-Hawaii Telescope (CFHT), Taiwan, Korea, Canada, France, and the CFHT, which is operated by the National Research Council (NRC) of Canada, the Institut National des Sciences de l'Univers of the Centre National de la Recherche Scientifique of France, and the University of Hawaii.

26 Several designations are used to denote MegaCam filters. For simplicity, we adopt $u^{*}, g, r, i$, and $z$, since of all the filters only $u^{*}$ is significantly different from the Sloan Digital Sky Survey's $u$.

27 Unless stated otherwise, magnitudes quoted in this paper are given in the AB magnitude system. See Appendix A for the numerical conversions between $\mathrm{AB}$ and Vega magnitudes for NGVS and NGVS-IR filters.
}

In particular, the identification and study of compact stellar systems and their stellar population properties are challenged by the age-metallicity-extinction degeneracy of optical colors (Worthey 1994; Arimoto 1996). The difficulties begin even at the level of sample definition: The distribution of old, metal-poor GCs merges into the parameter space of dwarf stars in optical color-color diagrams, while metal-rich GCs overlap with the locus of remote late-type galaxies. Around selected Virgo galaxies, the pointed observations of the Hubble Space Telescope (HST) ACS Virgo Cluster Survey have allowed a less ambiguous determination of the GC population thanks to the improved spatial resolution of HST (Côté et al. 2004; Jordán et al. 2009). Spectroscopic surveys are progressively extending the collection of well-classified objects to vaster areas, although with inevitable sparseness and depth limitations (e.g., Park et al. 2012; Romanowsky et al. 2012; Schuberth et al. 2012; Pota et al. 2013).

\subsection{The Near-Infrared Complement: NGVS-IR}

The NGVS near-infrared project (NGVS-IR) is designed to complement NGVS with deep, spatially well resolved images at wavelengths longer than the $z$ band to provide wider sampling of the spectral energy distribution (SED). The first part of this project, and the subject of this paper, is a deep $K_{s}$-band survey conducted with the wide-field infrared camera WIRCam (Puget et al. 2004) at CFHT that covers the central $2^{\circ} \times 2^{\circ}$ around the Virgo Cluster core, the location of the cD galaxy M87 and its vicinity. This area was the first fully surveyed in ugriz as part of the NGVS pilot survey, and we refer to this field as the pilot field hereafter. The NGVS-IR observations are currently being expanded beyond the pilot field with the $4 \mathrm{~m}$-class Visible and Infrared Survey Telescope for Astronomy (VISTA; Dalton et al. 2006) at the European Southern Observatory on Cerro Paranal in Chile. These observations slightly overlap with the pilot field and cover regions around the brightest elliptical galaxy in Virgo, M49, in the filters $J$ and $K_{s}$, as well as a strip of $J$ - and $K_{s}$-band images that connects the pilot field and the M49 field, and augments the coverage of the M86 Group. Taken altogether, the current NGVS-IR survey area provides a total contiguous spatial coverage of the central regions in Virgo of more than $\sim 20 \mathrm{deg}^{2}$.

\subsection{The Need for NGVS-IR}

The intermediate goals of the NGVS-IR survey are the development of a photometric selection method to identify GCs in the Virgo core region, the study of the age-metallicity-mass relations of GCs in M87 and the intracluster medium, the characterization of the stellar populations of ultracompact dwarf galaxies (UCDs) by combining optical and near-IR colors, the measurement of accurate distances to bright Virgo galaxies by using the surface brightness fluctuations method (Tonry et al. 1990), the study of the star formation histories and mass profiles of several Virgo galaxies, and, last, the determination of the galaxy mass function of the Virgo Cluster.

Although the Virgo Cluster has already been observed at near-IR wavelengths, none of the available near-IR surveys is deep enough to accomplish the NGVS science goals. The Two Micron All Sky Survey (2MASS; Skrutskie et al. 2006) provides uniform, precise photometry and astrometry over the entire celestial sphere in $J, H$, and $K_{s}$ bandpasses with $10 \sigma$ limiting magnitudes of 15.8, 15.1, and 14.3 mag, respectively. 2MASS offers the largest coverage of the Virgo Cluster in nearIR wavelengths, but its shallow photometry is not well suited for 
deep extragalactic studies. Deeper photometry is provided by the UKIRT Infrared Deep Sky Survey (UKIDSS; Lawrence et al. 2007) Large Area Survey, which has a much smaller area than 2MASS but reaches $5 \sigma$ limiting magnitudes of 20.5, 20.0, 18.8, and 18.4 mag in $Y, J, H$, and $K s$ bandpasses, respectively. The deepest near-IR photometry of Virgo galaxies corresponds to the Spectroscopic and $H$-band Imaging of Virgo survey (SHIVir; McDonald et al. 2011), which consists of pointed $H$-band observations with the University of Hawaii $2.2 \mathrm{~m}$ telescope, UKIRT, and CFHT in good seeing conditions $(<0$ '. 6$)$. While SHIVir provides deep $H$-band imaging for 286 VCC galaxies, it does not offer one-to-one contiguous mapping with the optical NGVS data as NGVS-IR does.

One of the most immediate aims of NGVS-IR is to improve source classification in order to obtain the cleanest possible samples of objects of any given type, and of GCs in particular. For this purpose, it was decided to produce a first set of mosaic images and catalogs with a focus on compact sources with small angular sizes. As shown below, adding near-IR photometry to the optical data provides an impressive separation of Virgo GCs from foreground stars and background galaxies in near-UV/optical/near-IR color space. The $u i K_{s}$ diagram, ${ }^{28}$ in particular, proves to be an exceptional tool for searches of GCs and compact stellar systems, such as UCDs (see Hilker 2011 and references therein), around galaxies in a large variety of environments. With more than $20 \mathrm{deg}^{2}$ of near-IR data soon available for Virgo, we will improve the decontamination of the optically selected samples currently analyzed within the NGVS collaboration and provide extensive studies of the GC distributions in luminosity, color, and space. Those distributions contain a record of GC formation mechanisms and GC system assembly histories. They are also a key test for stellar population models, which still struggle to exploit optical and near-IR photometry jointly (e.g., Pessev et al. 2008; Taylor et al. 2011; Riffel et al. 2011). Finally, the construction of clean GC samples facilitates very efficient spectroscopic follow-up campaigns with little contamination by other sources. This, again, turns GCs into probes of galaxy cluster dynamics out to large scales (e.g., Schuberth et al. 2012; Romanowsky et al. 2012; Pota et al. 2013)

A further motivation for NGVS-IR is the study of UCDs and the dwarf galaxy-GC transition. In the past decade, studies of the central regions of the Virgo and Fornax galaxy clusters have revealed dozens of massive compact objects in the magnitude range $-14 \lesssim M_{V} \lesssim-11$ with half-light radii between 10 and 30 pc (Haşegan et al. 2005; Jones et al. 2006; Misgeld \& Hilker 2011). Currently, two popular formation scenarios are that UCDs are the remnant nuclei of dwarf galaxies that were stripped by interactions (e.g., Bekki et al. 2003) and, alternatively, that they are agglomerates of multiple clusters that merged early in their lifetimes during violent star formation episodes such as those triggered by galaxy mergers (e.g., Fellhauer \& Kroupa 2002). These scenarios can be tested through studies of the mass-metallicity relation (e.g., Harris et al. 2006). NGVS will provide us with a larger sample of massive compact stellar systems within Virgo than ever before, and with NGVS-IR we will be able to quantify the ages, metallicities, and masses of these objects to higher accuracy than with optical colors alone (see, e.g., Puzia et al. 2002; Hempel \& Kissler-Patig 2004; Pessev et al. 2008).

\footnotetext{
${ }^{28}$ For simplicity, we will indifferently use $u$ and $u^{*}$ to refer to the MegaCam filter.
}

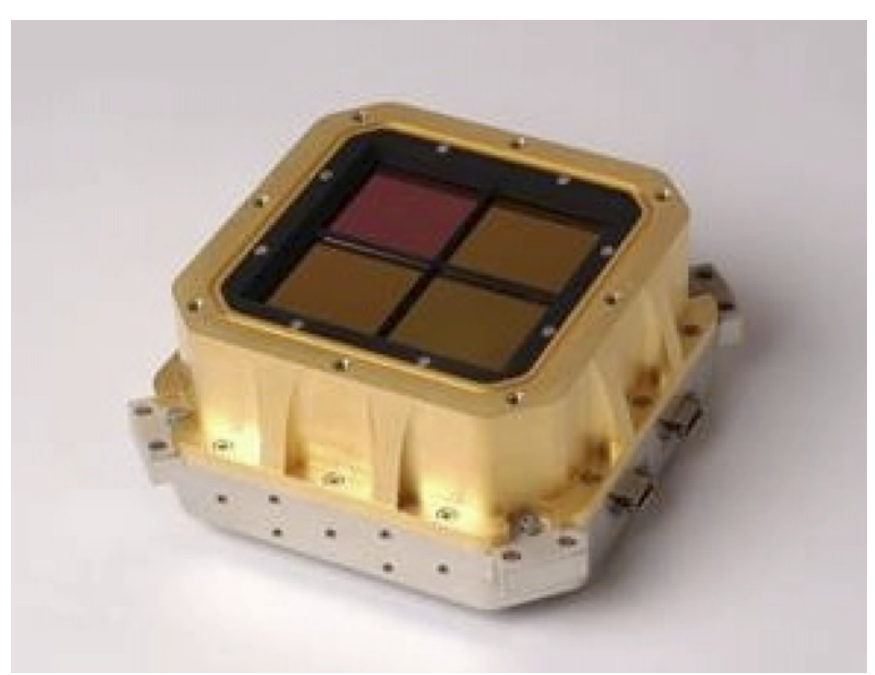

Figure 1. Illustration of the $2 \times 2$ mosaic WIRCam detector array. Each chip features a HAWAII-2RG near-IR detector with $18 \mu \mathrm{m}$ pixels. The patrol field has $2040 \times 2040$ active pixels with a 4 pixel wide border of reference pixels per chip. The field of view of the full detector array is about $21^{\prime} \times 21^{\prime}$ with $45^{\prime \prime}$ wide interchip gaps. Image courtesy of Academia Sinica Institute of Astronomy and Astrophysics (ASIAA).

(A color version of this figure is available in the online journal.)

The paper is organized as follows: We describe the details of our observations in Section 2 and the data reduction steps in Section 3. Sections 4 and 5 contain a discussion of the pointsource photometry and completeness estimates, while Section 6 includes the presentation of our first results. We summarize our work in Section 7.

\section{OBSERVATIONS}

\subsection{Instrumental Setup}

The NGVS-IR observations for the pilot field were carried out at CFHT using the WIRCam (Puget et al. 2004), which is mounted at the prime focus and consists of four cryogenically cooled $2048 \times 2048$ HAWAII-2RG near-IR $(0.9-2.4 \mu \mathrm{m})$ detectors arranged in a $2 \times 2$ mosaic, with typical interchip gaps of $45^{\prime \prime}$ and a plate scale of 0.3 pixel $^{-1}$ (see Figure 1). The array is operated at $\sim 80 \mathrm{~K}$ with negligible dark current $\left(\sim 0.05 e^{-}\right.$ $\mathrm{s}^{-1}$ ) and $30 e^{-}$readout noise. The field of view (FOV) of the full mosaic is about $21^{\prime} \times 21^{\prime}$ on the sky. The observations took place over a series of observing programs spanning the time period from 2009 December to 2010 July split over the French and Canadian time allocations (see Table 1 ). They are arranged in a mosaic of $6 \times 6$ WIRCam pointings, or tiles, that cover $2^{\circ} \times 2^{\circ}$ of the Virgo Cluster around the central giant elliptical galaxy M87 (see Figure 2) and match four pilot-project CFHT MegaCam pointings of the NGVS program (Ferrarese et al. 2012). All observations were carried out in Queued Service Observing mode at an air mass less than 1.2 under clear observing conditions, when the estimated seeing was better than 0 "' 8 .

\subsection{Dithering Pattern}

\subsubsection{General Considerations}

Pointed near-IR observations are usually executed with a dither pattern that sequentially observes the science target followed by a blank-sky region to account for the strongly varying near-IR sky surface brightness. This strategy involves significant overhead and generates many sky observations that 
Table 1

Journal of WIRCam Observations

\begin{tabular}{|c|c|c|c|c|c|c|c|}
\hline \multirow{2}{*}{$\begin{array}{l}\text { Program }^{\mathrm{a}} \\
\left(T_{\text {exp }}\right)\end{array}$} & \multirow[t]{2}{*}{ Tile } & \multirow{2}{*}{$\begin{array}{c}\text { R.A. } \\
\text { (J2000) }\end{array}$} & \multirow{2}{*}{$\begin{array}{c}\text { Decl. } \\
(\mathrm{J} 2000)\end{array}$} & \multicolumn{2}{|c|}{ Observing Date } & \multirow[t]{2}{*}{$N_{\mathrm{all}} \mathrm{c}$} & \multirow[t]{2}{*}{$N_{0.7} \mathrm{c}$} \\
\hline & & & & Month, Year & $\operatorname{MJD}(55 X X X)^{b}$ & & \\
\hline 09BC26 & $\mathrm{t} 1$ & $12: 33: 21$ & 11:40:09 & December, 2009 & 187,188 & 108 & 107 \\
\hline \multirow[t]{10}{*}{$(7.6 \mathrm{~h})$} & $\mathrm{t} 2$ & $12: 32: 04$ & $11: 40: 13$ & December, 2009 & 188,189 & 72 & 67 \\
\hline & $\mathrm{t} 3$ & $12: 30: 48$ & $11: 40: 14$ & December, 2009 & 194,195 & 109 & 81 \\
\hline & $\mathrm{t} 7$ & $12: 33: 21$ & 11:59:07 & December, 2009 & 190,191 & 106 & 104 \\
\hline & $\mathrm{t} 8$ & $12: 32: 05$ & 11:59:08 & December, 2009 & 187,188 & 109 & 103 \\
\hline & t9 & $12: 30: 48$ & $11: 59: 13$ & December, 2009 & 188,189 & 72 & 71 \\
\hline & $\mathrm{t} 10$ & $12: 29: 33$ & 11:59:09 & December, 2009 & 192,193 & 108 & 69 \\
\hline & $\mathrm{t} 11$ & $12: 28: 17$ & $11: 59: 14$ & December, 2009 & 194,195 & 100 & 69 \\
\hline & $\mathrm{t} 14$ & $12: 32: 05$ & 12:18:09 & December, 2009 & 190,191 & 108 & 108 \\
\hline & $\mathrm{t} 16$ & $12: 29: 33$ & $12: 18: 15$ & December, 2009 & 194,195 & 101 & 65 \\
\hline & $\mathrm{t} 22$ & $12: 29: 33$ & 12:37:09 & December, 2009 & 192,193 & 108 & 106 \\
\hline 09BF22 & $\mathrm{t} 4$ & $12: 29: 32$ & $11: 40: 13$ & December, 2009 & 191,193 & 72 & 71 \\
\hline \multirow[t]{3}{*}{$(1.5 \mathrm{~h})$} & $\mathrm{t} 17$ & $12: 28: 16$ & $12: 18: 13$ & December, 2009 & 191,193 & 72 & 72 \\
\hline & $\mathrm{t} 20$ & $12: 32: 05$ & $12: 37: 12$ & December, 2009 & 189 & 36 & 36 \\
\hline & $\mathrm{t} 31$ & $12: 33: 21$ & $13: 15: 12$ & December, 2009 & 189 & 36 & 32 \\
\hline $10 \mathrm{AC} 10$ & $\mathrm{t} 1$ & $12: 33: 21$ & $11: 40: 03$ & March, 2010 & 279,280 & 134 & 70 \\
\hline \multirow[t]{16}{*}{$(18.6 \mathrm{~h})$} & $\mathrm{t} 2$ & $12: 32: 05$ & $11: 40: 12$ & March-April, 2010 & $280,281,315$ & 112 & 39 \\
\hline & $\mathrm{t} 3$ & $12: 30: 49$ & $11: 40: 12$ & April, 2010 & $288,289,290,291,308,310$ & 112 & 96 \\
\hline & $\mathrm{t} 7$ & $12: 33: 21$ & 11:59:08 & March, 2010 & 283 & 108 & 108 \\
\hline & $\mathrm{t} 8$ & $12: 32: 04$ & $11: 59: 13$ & March, 2010 & 279,280 & 140 & 90 \\
\hline & t9 & $12: 30: 49$ & 11:59:09 & March-April, 2010 & $280,281,315$ & 109 & 32 \\
\hline & $\mathrm{t} 10$ & $12: 29: 33$ & 11:59:06 & March-April, 2010 & 283,287 & 116 & 71 \\
\hline & $\mathrm{t} 11$ & $12: 28: 17$ & 11:59:09 & April, 2010 & $288,289,290,291,308,310$ & 108 & 99 \\
\hline & $\mathrm{t} 13$ & $12: 33: 21$ & $12: 18: 10$ & April-July, 2010 & $310,315,342,374,377,378,379,380$ & 292 & 240 \\
\hline & $\mathrm{t} 14$ & $12: 32: 05$ & $12: 18: 08$ & March, 2010 & 283 & 108 & 108 \\
\hline & $\mathrm{t} 15$ & $12: 30: 49$ & $12: 18: 10$ & April-July, 2010 & $310,315,342,374,377,378,379,380$ & 301 & 196 \\
\hline & $\mathrm{t} 16$ & $12: 29: 33$ & $12: 18: 08$ & April, 2010 & $288,289,290,291,308,310$ & 108 & 86 \\
\hline & $\mathrm{t} 19$ & $12: 33: 21$ & $12: 37: 10$ & April-July, 2010 & $310,315,342,374,377,378,379,380$ & 296 & 247 \\
\hline & $\mathrm{t} 21$ & $12: 30: 49$ & $12: 37: 12$ & April-May, 2010 & $311,313,315,343$ & 175 & 86 \\
\hline & $\mathrm{t} 22$ & $12: 29: 32$ & $12: 37: 10$ & March-April, 2010 & 283,287 & 112 & 99 \\
\hline & $\mathrm{t} 25$ & $12: 33: 21$ & $12: 56: 12$ & April-May, 2010 & $311,313,315,343$ & 169 & 131 \\
\hline & $\mathrm{t} 26$ & $12: 32: 05$ & $12: 56: 12$ & April-May, 2010 & $311,313,315,343$ & 172 & 121 \\
\hline 10AF03 & $\mathrm{t} 4$ & $12: 29: 33$ & 11:40:08 & April, 2010 & $287,288,289$ & 113 & 108 \\
\hline \multirow[t]{16}{*}{$(13.4 \mathrm{~h})$} & $\mathrm{t} 12$ & $12: 27: 00$ & $11: 59: 15$ & April, 2010 & 289,290 & 158 & 88 \\
\hline & $\mathrm{t} 17$ & $12: 28: 17$ & $12: 18: 08$ & April, 2010 & $287,288,289$ & 112 & 108 \\
\hline & $\mathrm{t} 18$ & $12: 27: 01$ & $12: 18: 10$ & May, 2010 & $318,320,322,323$ & 117 & 87 \\
\hline & $\mathrm{t} 20$ & $12: 32: 04$ & $12: 37: 11$ & April-May, 2010 & $315,316,317$ & 130 & 97 \\
\hline & $\mathrm{t} 23$ & $12: 28: 17$ & $12: 37: 11$ & May-July, 2010 & 323,383 & 106 & 101 \\
\hline & $\mathrm{t} 24$ & $12: 27: 01$ & $12: 37: 15$ & April, 2010 & 289,290 & 153 & 117 \\
\hline & $\mathrm{t} 27$ & $12: 30: 49$ & $12: 56: 06$ & April, 2010 & 290,291 & 112 & 91 \\
\hline & $\mathrm{t} 28$ & $12: 29: 33$ & $12: 56: 08$ & April, 2010 & 290,291 & 108 & 95 \\
\hline & $\mathrm{t} 29$ & $12: 28: 17$ & $12: 56: 12$ & May-July, 2010 & 323,383 & 109 & 91 \\
\hline & $\mathrm{t} 30$ & $12: 27: 01$ & $12: 56: 07$ & May, 2010 & $318,320,322,323$ & 122 & 77 \\
\hline & $\mathrm{t} 31$ & $12: 33: 21$ & $13: 15: 07$ & April-May, 2010 & $315,316,317$ & 131 & 79 \\
\hline & $\mathrm{t} 32$ & $12: 32: 05$ & $13: 15: 17$ & May-July, 2010 & $341,343,382,383$ & 81 & 60 \\
\hline & $\mathrm{t} 33$ & $12: 30: 49$ & $13: 15: 07$ & May-July, 2010 & $341,343,382,383$ & 78 & 60 \\
\hline & $\mathrm{t} 34$ & $12: 29: 33$ & $13: 15: 10$ & May-July, 2010 & $341,343,382,383$ & 78 & 57 \\
\hline & $\mathrm{t} 35$ & $12: 28: 17$ & $13: 15: 08$ & May-July, 2010 & 323,383 & 109 & 99 \\
\hline & $\mathrm{t} 36$ & $12: 27: 01$ & $13: 15: 05$ & May, 2010 & $318,320,322,323$ & 116 & 78 \\
\hline
\end{tabular}

Notes. Grouped according to CFHT observing program.

a The total exposure time for the corresponding semester in hours is given below the program identifier.

b Modified Julian Date.

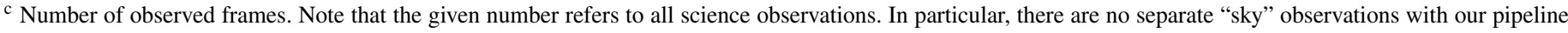
reduction technique (see Section 3.2.3).

${ }^{\mathrm{d}}$ Number of frames with seeing lower than $0{ }^{\prime \prime} 7$.

have generally no scientific use. In large surveys of noncrowded fields, one may use the area of interest itself to estimate the sky by implementing dithered observations. The dithering pattern also serves to fill the gaps between individual detector chips and defect areas of the detectors (of which WIRCam has quite a few). The NGVS-IR field, however, targets an area containing very extended galaxies. Surface brightness profile analyses of M87 found $D_{25} \approx 10^{\prime}$ (King 1978; de Vaucouleurs et al. 1991; Liu et al. 2005), which is similar to the FOV of a single WIRCam chip. We developed a dedicated dithering strategy to obtain sky 


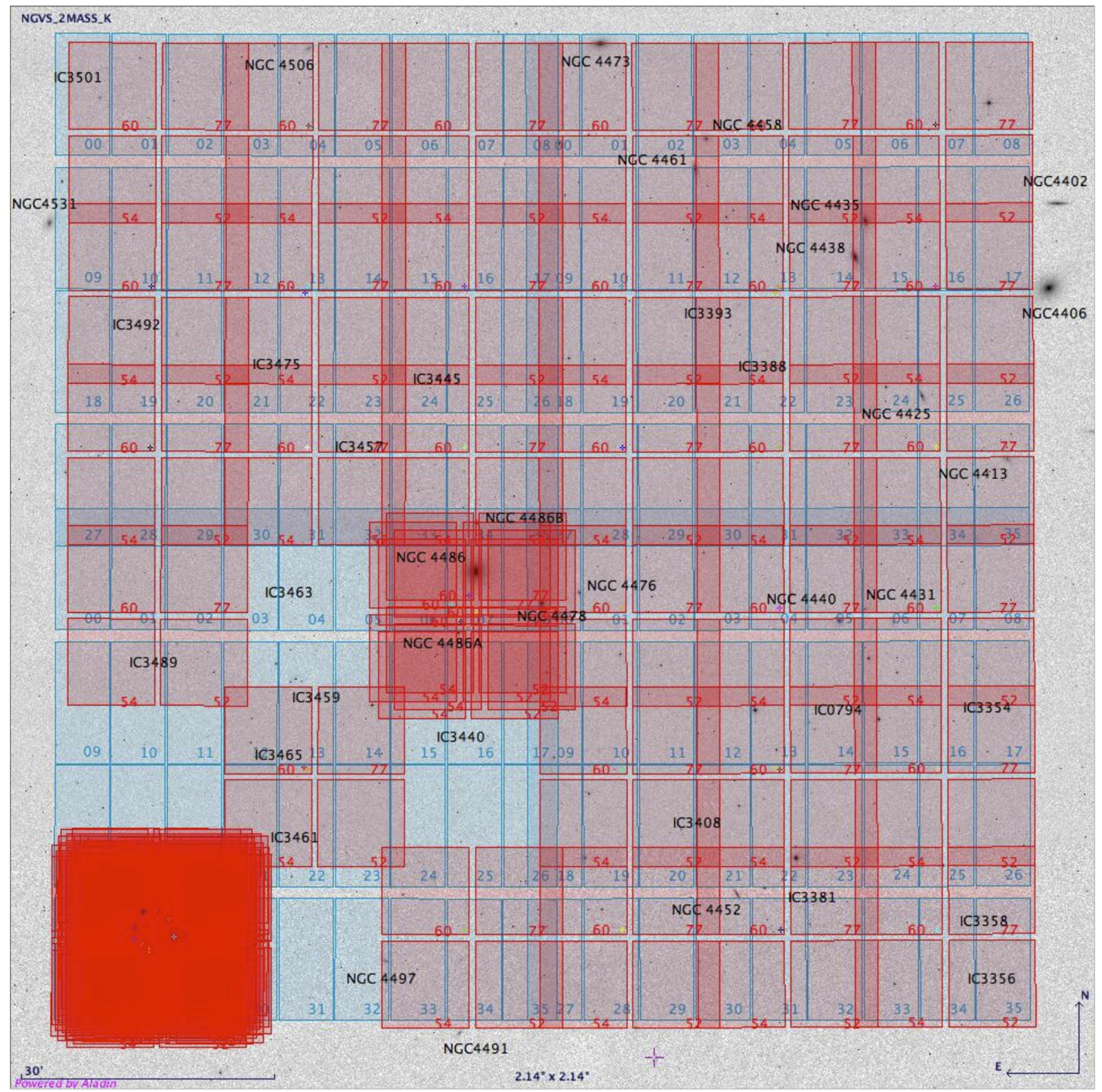

Figure 2. Illustration of the central $2.14 \times 2.14$ core region of the Virgo Cluster that was imaged by the NGVS-IR pilot project with WIRCam in a $6 \times 6$ pointing mosaic (red) and with MegaCam in a $2 \times 2$ pointing mosaic (blue). The bottom left tile of the WIRCam mosaic corresponds to tile 1 in Figure 3 and shows the coverage achieved with 27 visits (see Section 2.2.4 for details), while tile 15, overlapping the central giant elliptical galaxy M87 (NGC 4486 ), shows the coverage of an individual four-exposure dither (see Section 2.2.2). Note that four of the (red) WIRCam pointings in the lower left quadrant of the image, corresponding to tiles 2 , 7, 9, and 14 in Figure 3, have been deliberately left out for illustration purposes of the tile-to-tile overlap regions produced by the two interlocking dither types. All other WIRCam tiles show the sky coverage of one individual exposure. The most prominent galaxies are labeled with their corresponding NGC or IC numbers. The underlying image is a MASS $K$-band rendering of the field.

(A color version of this figure is available in the online journal.)

estimates within the survey area despite the presence of large galaxies. We use an individual integration time of $25 \mathrm{~s}$ to avoid saturation on the sky (the near-IR sky surface brightness on Mauna $\mathrm{Kea}^{29}$ varies around $\mu_{K_{\mathrm{s}}} \approx 16 \mathrm{AB}$ mag $\operatorname{arcsec}^{-2}$ ). A combination of small and large offsets, the latter of which are defined based on archival images of the field, are used to sample

${ }^{29}$ For a detailed description, see the CFHT WIRCam Web pages at http://www.cfht.hawaii.edu. the sky on a timescale shorter than the typical timescales of variations in its brightness.

\subsubsection{Exposure Dither}

Each visit to one of the 36 pointings, or tiles, of the survey area is implemented as a sequence of four dithered $25 \mathrm{~s}$ exposures. This exposure dither is illustrated in Figure 2 by the WIRCam tile footprint that overlaps NGC 4486 (M87). The exposure 


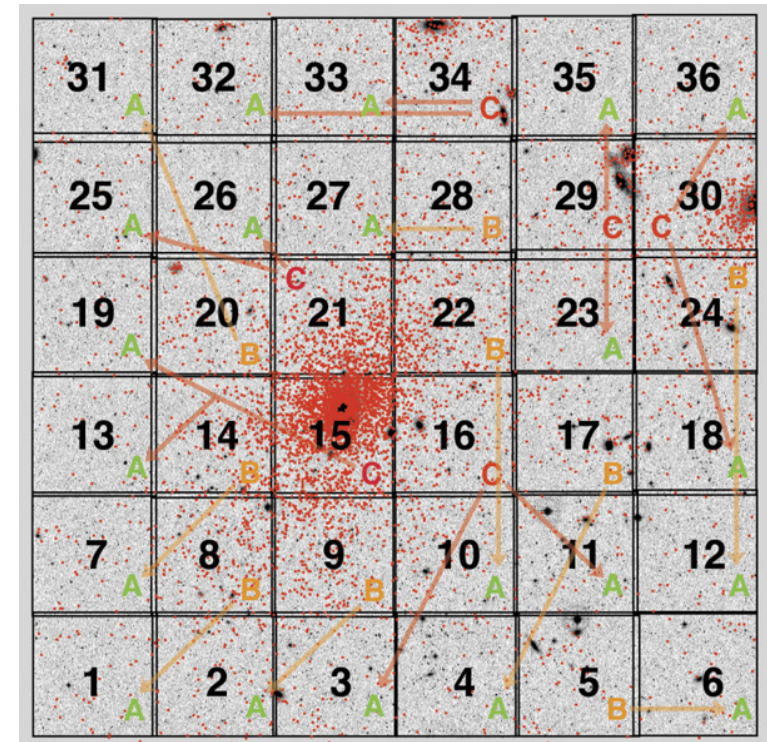

Figure 3. CFHT WIRCam pointing mosaic of the NGVS-IR pilot field that corresponds to Figure 2. The mosaic covers the inner $2^{\circ} \times 2^{\circ}$ around M87 with photometrically selected globular clusters (E. W. Peng et al. 2014, in preparation) shown as red dots on top of a $2 \mathrm{MASS}$ image. The arrows indicate the $\mathrm{A}_{1}-\mathrm{C}-\mathrm{A}_{2}$ and $\mathrm{A}-\mathrm{B}$ tile-to-tile sequencing of the observations.

(A color version of this figure is available in the online journal.)

dither pattern has a square geometry, but this square is tilted with respect to edges of the detector. This facilitates the process of neutralizing smaller chip defects and covering the $45^{\prime \prime}$ interchip gap, while keeping each subintegration safely in the linear regime of the detector. It also keeps individual visits to a given pointing reasonably short compared with usual timescales of sky surface brightness variations ( $\sim 10 \%$ in 10 minutes), so a given four-exposure visit to one tile may be used to estimate the sky in tiles visited just before or just after.

\subsubsection{Tile-to-tile Sequencing}

After one $4 \times 25 \mathrm{~s}$ visit is completed, we always move the telescope to a new pointing in the 36-tile mosaic of the WIRcam survey. How these large offsets are chosen is based on the relative crowding of the tiles. Figure 3 shows the mosaic overplotted on a $2 \mathrm{MASS}$ archive image, together with a sample of photometrically selected GCs indicated as red dots (E. W. Peng et al. 2014, in preparation). Each tile is assigned a category of $\mathrm{A}, \mathrm{B}$, or $\mathrm{C}$ based on the crowding and spatial extent of objects that it covers. We use two types of sequences: the $\mathrm{A}_{1}-\mathrm{C}-\mathrm{A}_{2}$ observing sequence combines the most crowded tiles (C-type) with two noncrowded tiles (A-type), while the shorter $\mathrm{A}-\mathrm{B}$ sequence combines one of the moderately crowded tiles (B-type) with one noncrowded tile (A-type). The arrows in Figure 3 illustrate which crowded $\mathrm{B}$ and $\mathrm{C}$ tiles are observed together in sequence with A tiles, which are relatively devoid of crowded regions and extended objects. This technique minimizes the required telescope slewing time and will allow accurate modeling of galaxy surface brightness profiles.

\subsubsection{Pointing Dither}

Each $\mathrm{A}-\mathrm{B}$ and $\mathrm{A}_{1}-\mathrm{C}-\mathrm{A}_{2}$ sequence is executed 27 times to reach the nominal exposure time of 45 minutes per pointing. We optimize the telescope nodding to simulate an on-tile dithering pattern. In other words, subsequent repeats of a given $\mathrm{A}-\mathrm{B}$ or $\mathrm{A}_{1}-\mathrm{C}-\mathrm{A}_{2}$ are offset. In contrast to the exposure dither (see above), we call this pattern the pointing dither. It guarantees that each time all pixels cover a different section of the sky, while the extent of the pointing dither allows us to combat the chip gaps and larger chip defects. The outer regions of the pointing dither pattern are covered by overlap regions between individual tiles of the entire $6 \times 6$ WIRCam mosaic.

An optimal dither pattern needs to be compact, be scalable, and prevent redundant pointings. Such a pattern is realized in nature by the arrangement of flower leaves (phyllotaxis) and seeds on flower heads, a prominent example, for instance, being the sunflower head. Mathematically, these patterns are described by a Fermat spiral (Vogel 1979). This is a special type of the Archimedean spiral and can be analytically expressed as $r=c n^{1 / \gamma}$, where $\gamma=2$ and $n=\theta / 137.508$, while $n$ is the index of the individual pointing. The value 137.508 is the golden angle. Figure 4 shows the arrangement of the 27 pointings we used for moving between repeated $\mathrm{A}_{1}-\mathrm{C}-\mathrm{A}_{2}$ or A-B sequences. We tested several Archimedean spirals with different scalings and found that the Fermat spiral yields the least pixel-to-pixel variance in terms of $x-y$ coordinate coverage (see the histograms in the left panel of Figure 4). In fact, the Fermat spiral pattern is the most compact and homogeneous coverage of a two-dimensional surface without $x-y$ pointing redundancy. We scaled it to the dimensions $(\Delta$ R.A., $\Delta$ decl. $)=\left(7^{\prime} .605,7^{\prime} .851\right)$ so that the central WIRCam interchip gap introduces the least variance on the final sky coverage map, which is illustrated in the right panel of Figure 4.

The pointing dither and the exposure dither $(4 \times 25 \mathrm{~s})$ work together to homogeneously fill the entire survey area, and we refer in the following to the full dither sequence as F2D27. The individual components of F2D27 are illustrated in Figure 2, where the exposure dither is shown for tile 15 (center of the field), while the pointing dither is depicted for tile 1 (lower left corner of the mosaic). Each tile in the WIRCam mosaic is offset by $19^{\prime}$ from its neighbor in $\Delta$ R.A. and $\Delta$ decl. to ensure enough overlap between the individual mosaic tiles, so that the sampling of the WIRCam interchip gap remains the most critical for homogeneous sky modeling, and similarly for the sampling of extended surface brightness structures, such as those of large galaxies. A coverage analysis of this WIRCam interchip gap region shows that our F2D27 pattern covers all sky locations more than 64 out of $4 \times 27=108$ times, while the vast majority of the interchip gap is covered at least 80 times. This translates into a signal-to-noise ratio fluctuation of only $14 \%$ and is fully propagated by our pipeline to the variance maps.

In summary, the observing strategy includes the following exposure-time outline for the two types of sequences:

$$
\begin{aligned}
\left(\mathrm{A}_{1}-\mathrm{C}-\mathrm{A}_{2}\right) & \rightarrow 3 \times 27 \times(4 \times 25) \mathrm{s}=8100 \mathrm{~s} \\
(\mathrm{~A}-\mathrm{B}) & \rightarrow 2 \cdot 27 \times(4 \times 25) \mathrm{s}=5400 \mathrm{~s}
\end{aligned}
$$

and contains a total of six $\mathrm{A}_{1}-\mathrm{C}-\mathrm{A}_{2}$ sequences and nine $\mathrm{A}-\mathrm{B}$ sequences that cover the entire field. Because of observing scheduling constraints, sequence A6-B5 could not be observed and thus is not discussed in this work. On the other hand, some of the observed sequences benefited from more than the requested 27 visits.

\section{DATA REDUCTION}

The data reduction process was divided into three major parts: the preprocessing stage, the main image processing, and the image postprocessing. 

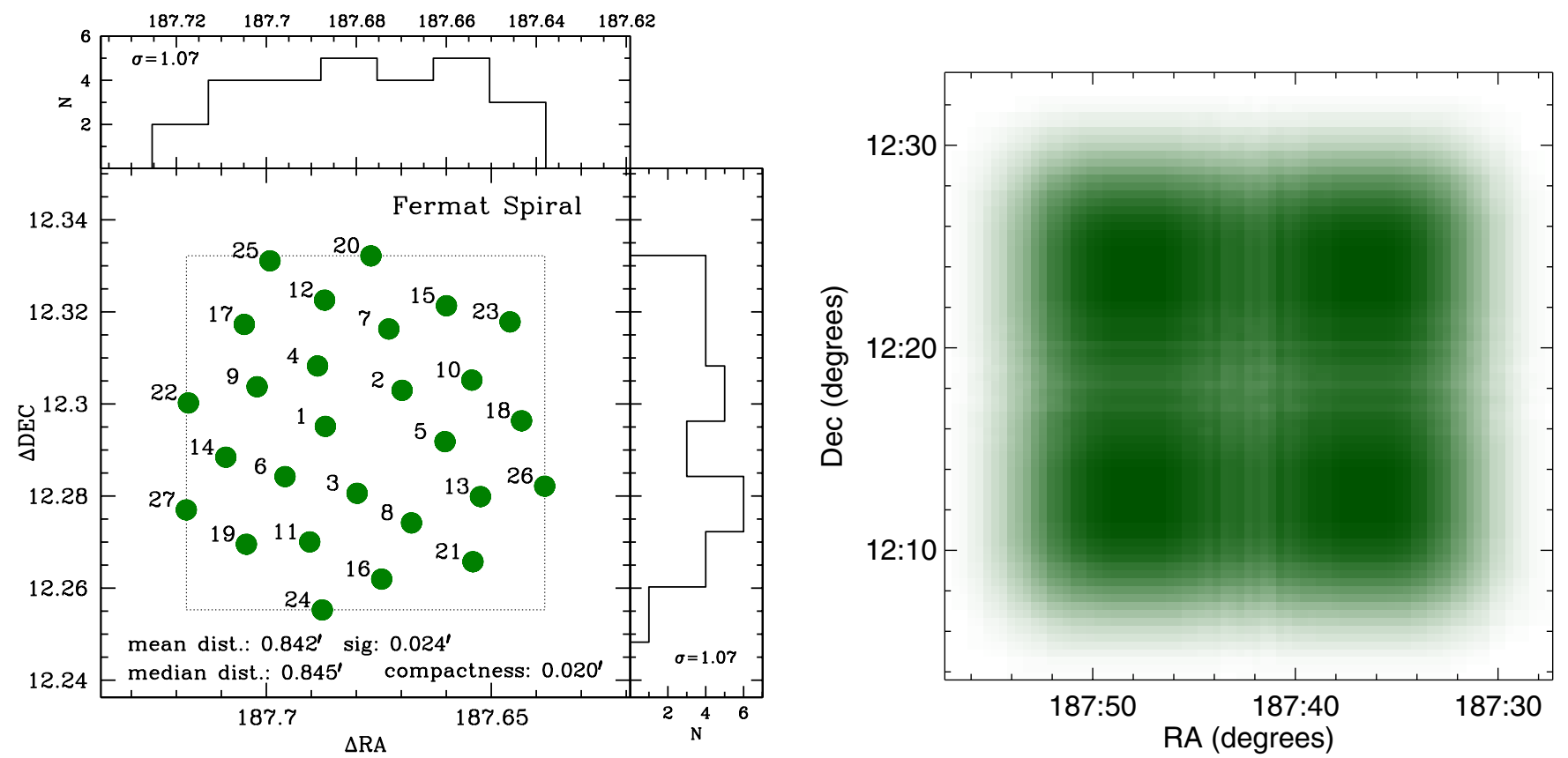

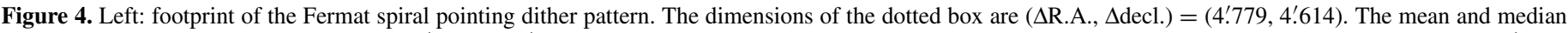

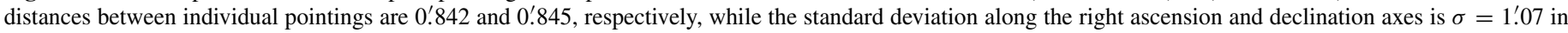

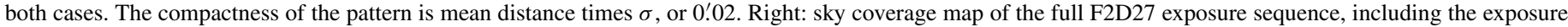

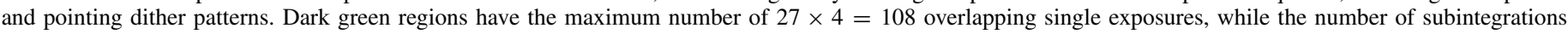
decreases toward the lighter green areas. The maximum variation in signal-to-noise ratio in the critical interchip region is only $14 \%$.

(A color version of this figure is available in the online journal.)

\subsection{Preprocessing}

The processing of the raw data was done using the "I'iwi pipeline, version 2.0. ${ }^{30}$ The pipeline involves the following steps: flagging saturated pixels, nonlinearity correction, dark subtraction, flat-fielding, and bad-pixel masking.

The saturated pixels were identified in the raw images using a threshold value of 36,000 ADU. A record of these pixels was kept, and they were flagged with the value 65535 in the final preprocessed image. The nonlinearity of the WIRCam detectors is about $5 \%$ at 30,000 ADU, and it was corrected at the beginning of the preprocessing. Dark frames were obtained at the beginning and the end of each observing night, and a master dark frame was computed by taking the median of 15 dark frames. The dark subtraction has a negligible impact on the preprocessed images, since the dark current of WIRCam detectors is significantly less than $1 e^{-} \mathrm{s}^{-1}$.

Twilight flat-fielding was applied by the 'I'iwi pipeline: Twilight flats are obtained at the beginning of each observing night, and a daily twilight flat is built by taking the median of 15 consecutive frames. The pipeline divides the semester into several sets of observing nights and then computes a master twilight flat on each set. All science images were corrected by dividing by the corresponding normalized master flat.

The master bad-pixel masks were built by analyzing the normalized master flats previously computed. Bad pixels were identified by applying a sigma-threshold algorithm. The badpixel masks were constructed to have a value of 1 for good pixels and 0 for bad pixels.

We refer to the preprocessed images in the following as science images.

\footnotetext{
${ }^{30}$ See http://www.cfht.hawaii.edu/Instruments/Imaging/WIRCam/ IiwiVersion2Doc.html.
}

\subsection{Main Image Processing}

The processing consisted of masking cosmic rays and satellite trails in the science images, removing the residuals left by saturated stars, subtracting the sky from the images, computing the astrometric solution, applying the photometric calibration, and producing the final stacked images.

\subsubsection{Cosmic-ray and Satellite Trail Removal}

The cosmic rays were identified by running the Laplacian cosmic-ray identification algorithm L.A.Cosmic (van Dokkum 2001) on the science images. This algorithm is based on a variation of the Laplacian edge detection method, and cosmic rays are identified by the sharpness of their edges. The code convolves the image with a Laplacian kernel, identifies cosmic rays as sharp borders in the image, and creates a cosmic-ray mask for each science image. The best L.A.Cosmic parameters were obtained by performing a visual inspection of the masks and verifying that the peaks of nonsaturated bright stars were not misidentified as cosmic rays.

The detection of satellite tracks is not possible in the preprocessed images, since the sky brightness dominates the image while a typical satellite track is 100 times fainter than the sky. We ran a quick sky removal on each image (target) that consisted of identifying the 10 closest images in observing time (sky) and then taking the median of the sky images pixel by pixel and subtracting it from the target image. The sky-subtracted images were smoothed with a boxcar average of width 20 pixels, and the coordinates of pixels with signal-to-noise ratio greater than 1.2 were registered. Satellite tracks were identified as straight lines in the $x-y$ detector plane of the registered coordinates. 


\subsubsection{Saturation Correction}

Bright and saturated stars leave residuals in the science images that last for about six subsequent images. First, the saturated bright stars were identified as those regions of at least 4 connected pixels with counts higher than 65535 and not masked as bad pixels in the master bad-pixel mask. For chips 1 and 3, it was necessary to mask the subsequent three images, while for chips 2 and 4 it was necessary to mask the subsequent six images because of the different chip and detector characteristics. The science images were sorted according to their observing time, the pixels belonging to a saturated star were identified, and the six (or three) consecutive images after saturation were masked at the corresponding coordinates.

\subsubsection{Sky Subtraction}

One motivation for the $\mathrm{A}_{1}-\mathrm{C}-\mathrm{A}_{2}$ or $\mathrm{A}-\mathrm{B}$ observational strategy presented in Section 2.2 was to design a high-quality sky subtraction method. The observations in each $\mathrm{A}_{1}-\mathrm{C}-\mathrm{A}_{2}$ or $\mathrm{A}-\mathrm{B}$ sequence were declared to be target or sky tiles, and for each target image there was a set of sky images defined for computing and subtracting the sky in the corresponding target image. The sky images from A-type tiles are generally less crowded regions than target images but do still contain a significant number of pointlike and extended sources that need to be masked before computing the final sky image. The sky subtraction method is a two-step process: the first iteration consists of computing a median sky image for each target image, subtracting it from the target image, and finally building a stacked target image from all such treated frames in a sequence; the second iteration consists of identifying the sources in the stacked target image, masking these sources in the each individual sky image, computing a new median sky, and finally subtracting it from the target image.

The selection of the set of sky images to model and subtract the sky contribution in each target image depends on the amplitude and variance of the sky surface brightness and is based on the following three criteria:

1. The category of the $A_{1}-C-A_{2}$ or $A-B$ observing sequence that the target image is part of;

2. The time difference between sky images and the targetimage time stamp; and

3. The need for a sufficient number of sky images to compute a reliable median sky image.

The first criterion is related to the sky-target dithering sequence explained in Section 2.2.3 and consists of the following set of rules: $(a)$ if the science image belongs to type A tile, then sky images are selected from type A tiles of the same sequence; (b) if the science image belongs to a type B tile, then sky images can be selected from type A and type B tiles; $(c)$ if the science image belongs to a type $\mathrm{C}$ tile, then sky images can be selected only from type A tiles.

The second criterion consists of choosing sky images close enough in time to the target image. We used only those sky images taken $\leqslant 15$ minutes before or after the corresponding target-image observation. In particular, at the beginning and the end of each observing run there were sets of sky images containing just eight images, and as will be explained by the third criterion, that is below the recommended number of frames to model the sky.

The third criterion consists of defining a sufficient number of sky images to compute a reliable median sky image that robustly captures the sky variations. We ran several quality tests on the sky images, and the conclusion was that the minimum number of frames for this survey is 10 sky images, given the variability of the near-IR sky throughout our observing campaign. This criterion was applied as follows: we sorted the sky images according to their time stamp with respect to the target image, then we computed the maximum between 10 and the number of sky frames that fulfilled the first and second criteria, and the final set of sky images was defined by applying that maximum number.

Once the set of sky images is defined for each target image, we compute the pixel-to-pixel median sky image and subtract it from the corresponding target image.

\subsection{Postprocessing}

We applied postprocessing routines to the images because some of the processed images still showed visible systematics. These postprocessing steps consist of removing the slope from each detector amplifier and subtracting large-scale variations of the sky background.

\subsubsection{Residual Amplifier Differences}

Each WIRCam detector has 32 amplifiers with different gains, which result in background-level offsets that differ by $\sim 10 \%$ in the twilight flat-field images. Most of the signal of the amplifiers disappears after flat-field correction and removing the sky during the preprocessing pass, but there is still a nonnegligible contribution that appears as horizontal bands through the image. To model and subtract this residual, we start with sky-subtracted images obtained from the processing pass and select the pixels belonging to each amplifier, taking the median along the $x$-axis of the detector. Then we fit the variation with a linear relation as a function of the $y$-axis coordinate and finally subtract this model from each horizontal band in each of the sky-subtracted images.

\subsubsection{Large-scale Variations}

After close examination of the gain-corrected and skysubtracted images, we found large-scale variations of the image background still present after the sky removal, with an amplitude of $\sim 0.3 \%$ of the typical sky. These features are likely produced by a variation in the thermal stability of the detector, internal reflections within the instrument due to the presence of large galaxies, or both. They have a nonnegligible effect on the stacked the images.

In order to improve the background flatness, we mask the sources and model the large-scale background structure in each frame. For this purpose, we first use SWarp (version 2.19; Bertin et al. 2002) to build a stacked image using all the sky-subtracted frames computed in the processing stage. We run SExtractor on the stacked images (version 2.5.0; Bertin \& Arnouts 1996) and obtain the segmentation maps with pixels associated with the sources. To remove the outer low surface brightness parts of galaxies, we increase the sizes of the masked regions by $50-800$ pixels, depending on the size of the galaxy, and project the source mask back into the individual prestack sky-subtracted images. The large-scale variations are computed on each of the four WIRCam detectors independently. Each detector is divided into a grid of $16 \times 16$ cells. Those cells in which more than $50 \%$ of the pixels are masked are discarded. In all other cells, a median background value is computed. A large-scale variation image is then computed using the kriging linear interpolation algorithm as implemented in IDL (version 8). This background model is then subtracted to produce the final science-grade images. This 
method has some limitations when dealing with galaxies with extremely extended surface brightness profiles. However, these limitations have no impact on the analyses and results presented in this paper.

\subsection{Astrometric Calibration}

We calculate the relative astrometric solution with the SCAMP software package (version 1.7; Bertin 2006), which reads the catalogs produced by SExtractor and cross-matches the source positions against an astrometric reference catalog such as 2MASS (Skrutskie et al. 2006). We ran SCAMP using a maximum search range of $\sim 0$ '.15 (POSITION_MAXERR $=0.15$ ) and a minimum signal-to-noise ratio of 40 (SN_THRESHOLDS $=40,80)$ to match reference stars.

We computed the distortion maps for each of the WIRCam detector chips and find that the pixel scale varies in a radially symmetric way by at most $0.5 \%$ between the center of the $2 \times 2$ detector mosaic and the outer radius of the WIRCam FOV. The astrometric calibration against the 2MASS reference frame is based on $\sim 10^{3}$ stars, and the resulting astrometric world coordinate solution accuracy has an approximately Gaussian distribution with a full width at half-maximum smaller than 0.'02.

\subsection{Photometric Calibration}

We used the 2MASS Point Source Catalog (Cutri et al. 2003) to perform the preliminary photometric calibration of the skysubtracted images. We selected 2MASS pointlike sources with photometric quality flags of A and B (i.e., reliable photometry), which correspond to sources with signal-to-noise ratio greater than 7 and magnitude error less than $0.16 \mathrm{mag}$.

The photometric calibration was done using SCAMP, as for the astrometric calibration. SCAMP distinguishes between two types of exposures: those observed under photometric conditions, and the others. The information about the photometric nights was obtained from the Queued Service Observing weather reports, and the images observed during those nights were visually inspected to define a clean sample of exposures taken in photometric conditions. The zero point of the photometric calibration was computed using

$$
m_{\mathrm{std}}=m_{\mathrm{inst}}+z p-k X
$$

where $m_{\text {std }}$ is the 2MASS magnitude, $m_{\text {inst }}$ is the instrumental magnitude measured on the sky-subtracted images, $z p$ is the zero point of the photometric calibration, $k$ is the air-mass term, and $X$ is the air mass of the observation.

The four WIRCam detectors have different gains, and in principle a $z p$ should be computed per exposure and detector, but the number of reliable 2MASS stars per detector can be as low as 10. We opted for computing the $z p$-offsets between the WIRCam detectors using groups of exposures and then keeping these offsets fixed to assess variations in $z p$ between exposures in the group. The $z p$-offsets are computed by grouping the exposures every 15 minutes and then cross-matching the detected sources with reliable $2 \mathrm{MASS}$ pointlike sources and computing the median value of $m_{\text {std }}-m_{\text {inst }}$ for all the stars on each detector. Figure 5 shows the distribution of the difference between the $z p$ values of chip 1 and those of chips 2, 3, and 4 (zp offset), where the distribution for chip 2 is found to be the most concentrated. The $z p$ offsets for detectors 2,3 , and 4 are $0.12,0.08$, and 0.03 mag, respectively.

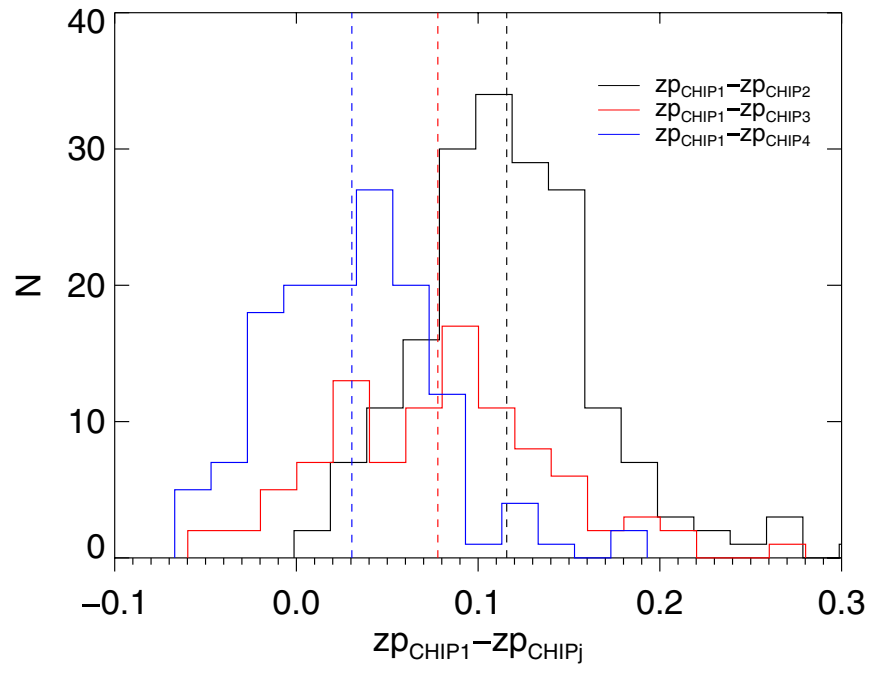

Figure 5. Distribution of the differences between the zero points of detector 1 and detectors 2, 3, and 4. Chips 2 and 4 show a peaked distribution, and chip 3 a broader distribution. The average zero-point offsets with respect to chip 1 are $0.12,0.08$, and 0.03 mag for chips 2,3 , and 4 , respectively.

(A color version of this figure is available in the online journal.)

To compute the air-mass term $(k)$ in Equation (1), we first compute the mean value of $m_{\text {std }}-m_{\text {inst }}$ on each exposure and then apply a linear regression analysis using only photometric nights. The estimated value of $k$ for this survey is $k=0.041$. We use Equation (1) to estimate the $z p$ for exposures observed on photometric nights and add the value as PHOT_ZP to the header.

For exposures observed on nonphotometric nights, SCAMP runs an internal cross-match of pointlike sources and then adjusts the $z p$ for those exposures by minimizing Equation (2). We adopt the same parameters as in the previous section, but this time we use a signal-to-noise ratio of 20 or higher in order to have a larger sample of stars for the photometric calibration $($ SN_THRESHOLDS $=20)$. We compute

$$
\chi^{2}=\sum_{s} \sum_{a} \sum_{b>a} w_{a b}\left(z p_{a}+m_{\mathrm{inst}, a}-z p_{b}+m_{\mathrm{inst}, b}\right)^{2},
$$

where $a$ and $b$ refer to exposures that contain the pointlike source $s$ in the overlapping region and $w_{\mathrm{ab}}$ is the nonzero weight for the pair of detections in exposures $a$ and $b$, which is computed as $w_{a b}=1 / \sigma_{\text {phot }}^{2}$, where $\sigma_{\text {phot }}^{2}$ is the square sum of all contributing photometric variances.

The instrumental magnitude, $m_{\text {inst }}$, in Equation (1) is measured on the sky-subtracted images with circular-aperture photometry of radius $4 \times \overline{\mathrm{FWHM}}$, where $\overline{\mathrm{FWHM}}$ is the mean value of the full width at half-maximum of the bright stars in each exposure. Figure 6 shows the distribution of the FWHM of pointlike sources of all the images in the survey. The FWHM distribution is not homogeneous and covers a range between 0.4 and 1".0. Thus, the photometry aperture is large enough to include more than $95 \%$ of the total light. The photometric zero points adopted in this work are shown in Table 2 . The $\overline{z p}_{K}$ refers to the mean zero point of science frames observed on the respective night and the quoted uncertainty to its standard deviation.

\subsection{Stacking and Data Quality Assessment}

The sky-subtracted images were stacked using SWarp (version 2.19; Bertin et al. 2002) after the astrometric and photometric calibrations were completed. SWarp uses the astrometric 


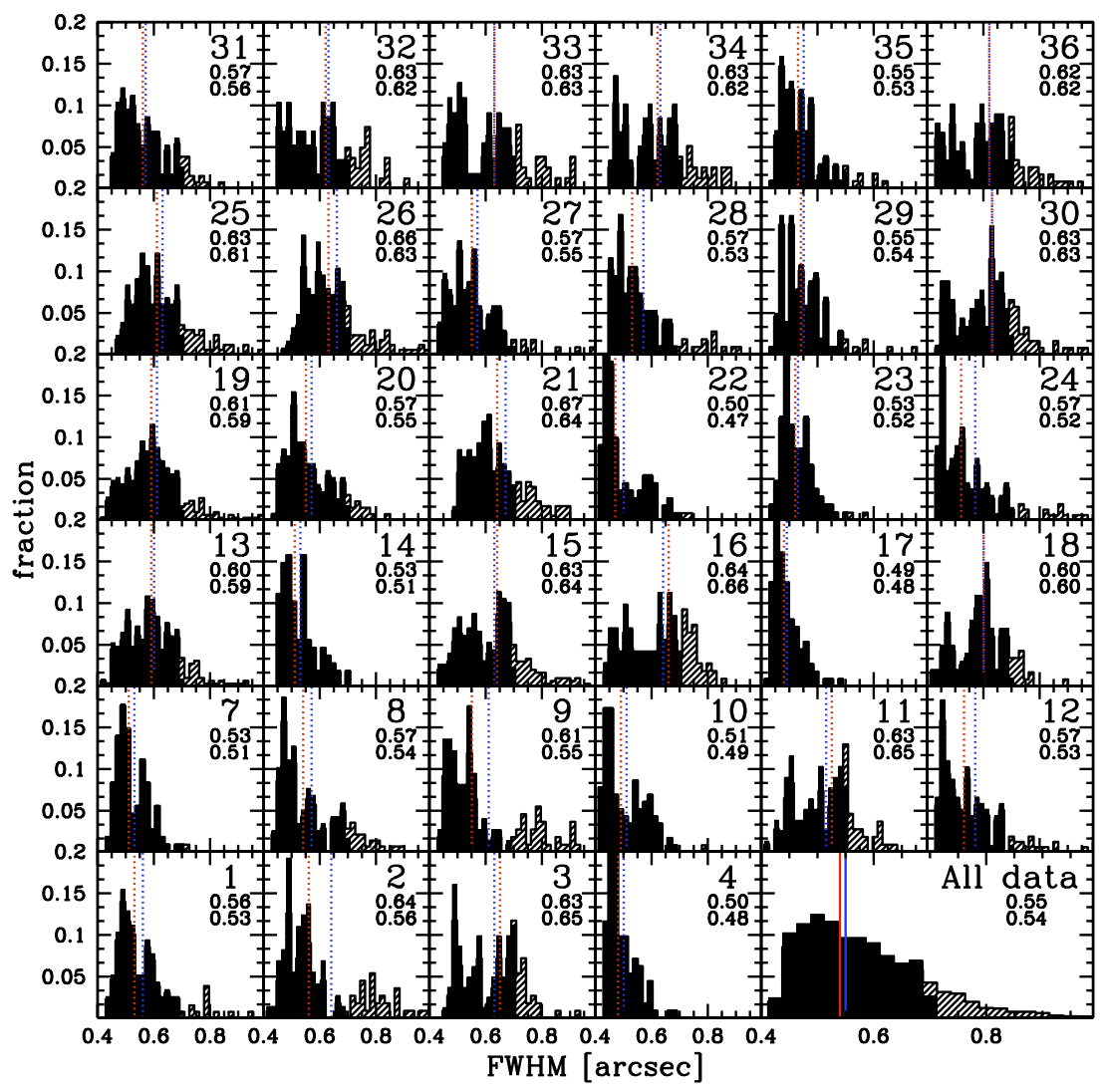

Figure 6. Seeing-quality distributions of all images (hatched histograms) for each NGVS-IR pilot-field tile that contributed to the final mosaic (filled histograms). The tile number is given at upper right in each panel, as well as the mean (upper value) and median (lower value) of each distribution, considering all frames available for each tile. The mean and median values are marked by blue and red vertical dotted lines, respectively. Note that only images with seeing better than 0 '! 7 were used in this work, marked by the filled histograms. In the bottom right panel (recall that no data were obtained for tiles 5 and 6 ), we illustrate the seeing distribution of all images of the NGVS-IR pilot field and provide the corresponding mean and median values of only the images with seeing better than 0!'7, which are shown by solid vertical lines. (A color version of this figure is available in the online journal.)

solution calculated by SCAMP and combines several frames to produce a final image of a given size and pixel scale. We use the $i$-band quadrants of the NGVS pilot-field area as a reference for building same-sized FOV $K_{s}$-band stacked images, since it helps the subsequent analysis. We note that the pixel scale of WIRCam images is $0^{\prime} .3$, while the pixel size of our final stacked images is $0{ }^{\prime} 186$ and that we build the stacked images using only images with FWHM smaller than 0.7 (see Figure 6). The median seeing of all selected data is $0{ }^{\prime \prime} 54$. The NGVS-IR pilot field covers a total area of $3.98 \mathrm{deg}^{2}$ and consists of 34 slightly overlapping tiles with coverage of about $26.5 \times 26^{\prime} .5$. For datahandling purposes, we subdivide the pilot-field area into four quadrants roughly corresponding to about $1 \mathrm{deg}^{2}$ each.

The large majority of the sky-subtracted images in the survey are supercritically sampled (above the Nyquist limit). We use the Lanczos- 2 resampling algorithm, which offers a good compromise between improving the image resolution and minimizing the number of artifacts introduced by the resampling routine. The images are combined using a sigma-clipping algorithm that computes the dispersion on a pixel-by-pixel basis and rejects pixels beyond $\pm 3 \sigma$ of the mean value.

The NGVS-IR image stacks, which this paper is based on, reach $K_{s}$-band surface brightnesses fainter than $\mu_{K_{s}} \approx 24 \mathrm{AB}$ mag $\operatorname{arcsec}^{-2}$. In some of our NGVS-IR stacks, we can make out dwarf galaxies with $\mu_{K} \approx 24.4 \mathrm{AB}$ mag $\operatorname{arcsec}^{-2}$. The extended emission of $94 \%$ of the known VCC galaxies can be visually seen in the final images. However, we emphasize that the data products discussed here were not obtained with the purpose of optimizing surface brightness sensitivity, and we therefore postpone any study of luminosity profiles to future work. Specific data reduction procedures will also be required for the study of near-IR surface brightness fluctuations in Virgo galaxies. In Figure 7, we illustrate the data quality with a color stack of an $11^{\prime} \times 11^{\prime}$ cutout around the interacting galaxy pair NGC $4435+4438$, which includes five of the faintest VCC dwarf galaxies and even fainter low surface brightness dwarfs, all of which are detected on the $K_{s}$ stacks.

Before performing detailed completeness tests, we note that visual inspection of the images shows that our NGVS-IR WIRCam survey achieves a $K_{s}$ point-source sensitivity of $\sim 23 \mathrm{mag}$, thus reaching about 2.9 mag deeper in this passband than the UKIDSS Large Area Survey, the most extensive and deepest recent survey covering the NGVS pilot field (Lawrence et al. 2007, 2012). NGVS-IR compares in surface brightness sensitivity with the pointed $H$-band observations of the SHIVir survey $^{31}$ of bright Virgo galaxies of McDonald et al. (2011). These, however, are not contiguous images and were taken with several instruments in varying observing conditions.

\section{PHOTOMETRY}

Source detection in the $K_{s}$ stacks and photometric measurements were performed with SExtractor (version 2.5.0; Bertin \& Arnouts 1996) and PSFex (Bertin 2011). After a first SExtractor run for source detection, PSFex produces local models of the

\footnotetext{
31 See http://www.astro.queensu.ca/virgo/.
} 


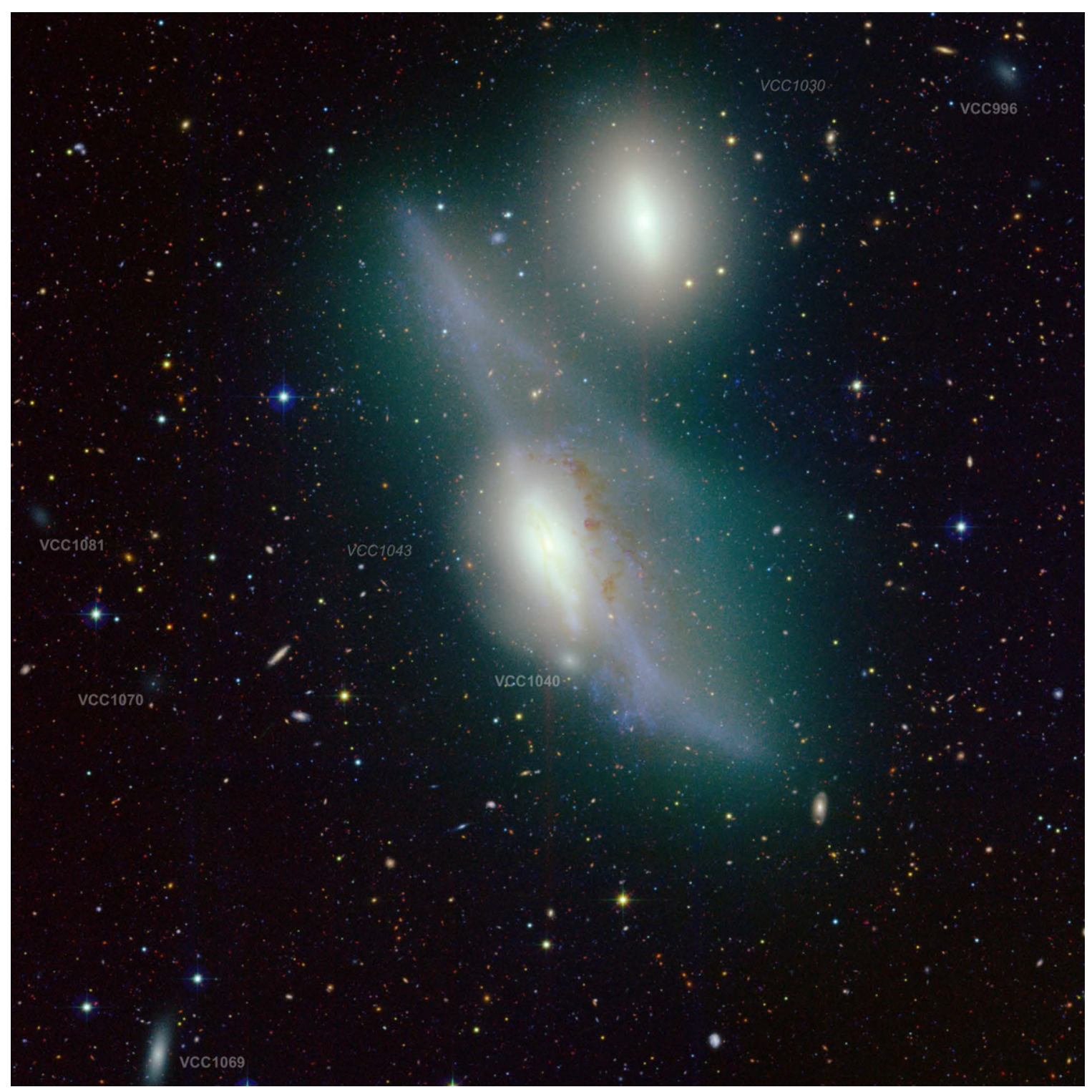

Figure 7. Sample color composite from the combined NGVS + NGVS-IR data set of a $11^{\prime} \times 11^{\prime}\left(53 \times 53 \mathrm{kpc}^{2}\right)$ sample FOV around the merger remnant NGC 4438 (VCC 1043; center of the image) and its bright companion NGC 4435 (VCC 1030; see also Figure 2). The image is an RGB composite using the $u^{*}$ (blue), $i$ (green), and $K_{s}$ (red) data and a logarithmic luminosity stretch. Besides the impressively detailed substructure in the merger remnant, numerous low surface brightness dwarf galaxies are visible. We label five VCC dwarf galaxies, which are among the faintest members of the VCC and are all clearly detected in our $K_{s}$-band images. Note that the vast majority of sources with small angular sizes are red background galaxies. This extremely deep near-UV/optical/near-IR photometry is well suited for finding galaxy clusters in the redshift range $0.5 \leqslant z \leqslant 1$ that are in the process of formation.

(A color version of this figure is available in the online journal.)

point-spread function (PSF), which can then be used in a second run of SExtractor to obtain integrated PSF magnitudes of stars. As input for PSFex, we use a clean sample of stellar sources, obtained by combining a compactness and a color criterion (see Section 6.3), and by rejecting any star that saturated the detector in one or more individual images that entered the stack. The PSF was modeled separately across the four individual quadrants of the pilot field (see Figure 8). Each of these quadrants consists of $3 \times 3$ WIRCam pointings, and PSF variations are dominated by pointing-to-pointing variations in the corresponding FWHM distribution functions (see Figure 6). The spatial variations of the PSF are modeled with polynomials of degree 7 to allow for changes on the typical scale resulting from this pattern.

With the SExtractor parameters DETECT_THRESH $=1.4$, ANALYSIS_THREST $=2.0$, DETECT_MINAREA $=6$, and
DEBLEND_MINCONT $=0.000001$, a total of $\sim 450,000$ sources are detected, and about 17,800 of these are identified as stars. The accuracy of our measurements are best described by focusing on these point sources. In Figure 9, we compare the WIRCam $K_{s}$ photometry with the corresponding 2MASS and UKIDSS data. The dispersion in both panels is predominantly due to the photometric errors in 2MASS and UKIDSS. The UKIDSS data (DR8; Lawrence et al. 2007) provide a tighter calibration relation than 2 MASS. Both panels exhibit a significant color term. The left panel of Figure 10 shows that the color term relating WIRCam and UKIDSS magnitudes seems to depend on the $K_{s}$-band magnitude. At the bright end, one can see the actual effect of the different shapes of the $K_{s}$ filters of WIRCam and UKIDSS, while at the faint end the slope mostly reflects large uncertainties in the UKIDSS $K_{s}$ photometry. For the UKIDSS native magnitudes (in the Vega photometric 

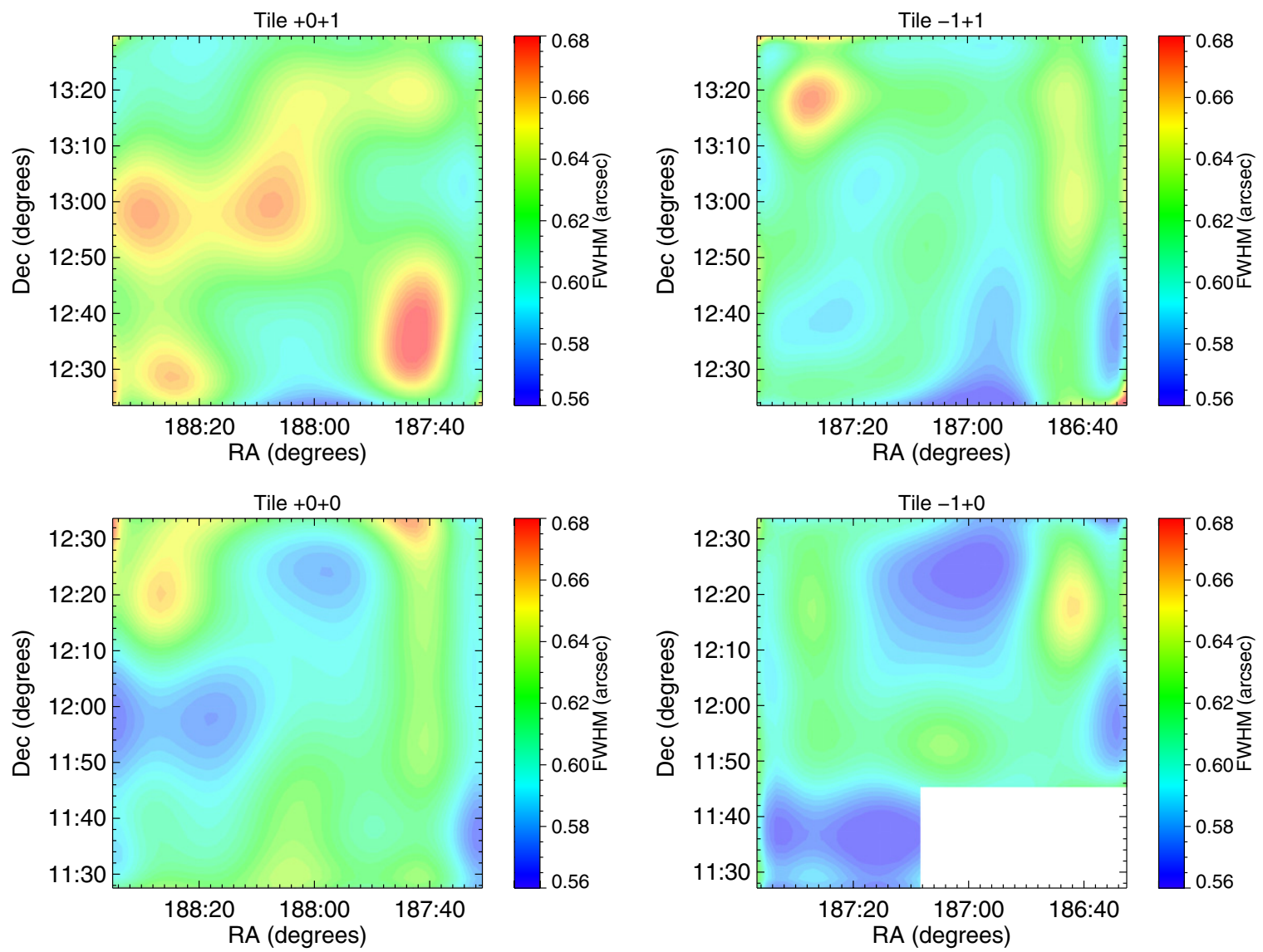

Figure 8. Image-quality maps for the NGVS-IR pilot field split into the four quadrants corresponding to the NGVS MegaCam tiles $+0+0,+1+0,+1+1$, and $+0+1$ (see Ferrarese et al. 2012 for details). The color bars parameterize the FWHM of pointlike sources in units of arcseconds over the survey area, the maximum variation of which is $\sim 20 \%$, which is mainly due to the seeing distributions of observations in each tile of the NGVS-IR mosaic as illustrated in Figure 6.

(A color version of this figure is available in the online journal.)
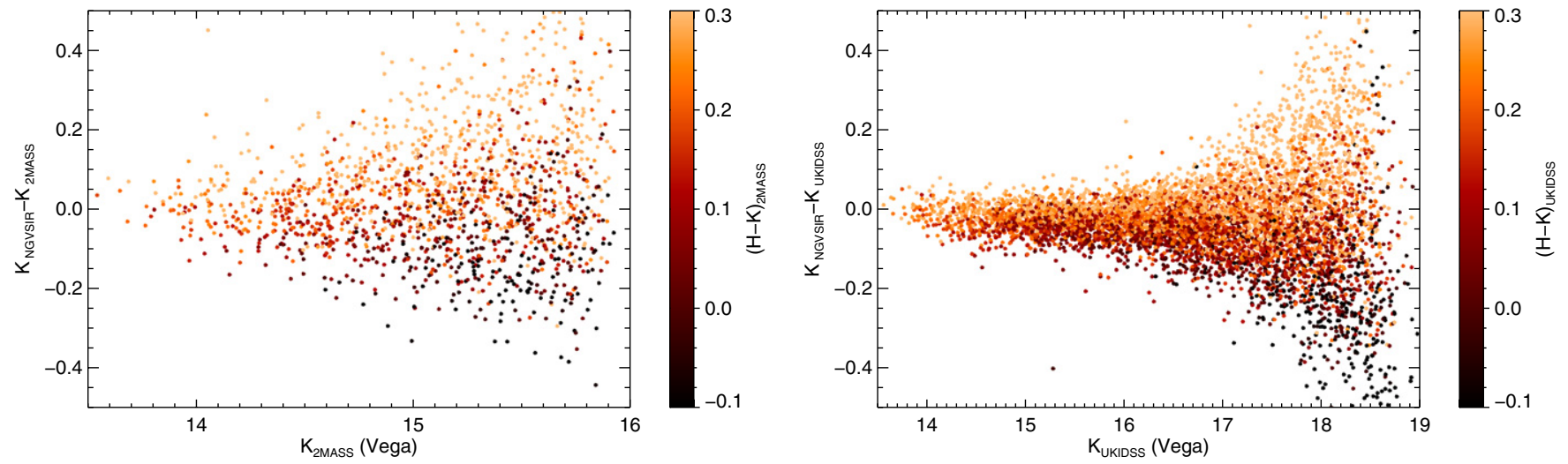

Figure 9. Photometric calibration accuracy of the NGVS-IR pilot-field data versus 2MASS (left) and UKIDSS (right) data. Objects in these plots are point sources according to their $K_{s}$-band morphology and NGVS/NGVS-IR colors and match coordinates in UKIDSS or 2MASS data to better than $1^{\prime \prime}$. The right color bar encodes the $H-K$ color measured in the corresponding comparison system and illustrates significant residual color terms in the photometric calibration. To avoid conversion of UKIDSS and 2MASS magnitudes into the AB system, the plots show point-source magnitudes in the Vega magnitude system. Note the significantly different photometric depth (i.e., $\sim 3 \mathrm{mag}$ ) between the 2MASS and UKIDSS data. The data distributions in both panels are entirely dominated by the photometric uncertainties of the 2MASS and UKIDSS data.

(A color version of this figure is available in the online journal.)

system; see Appendix A), we have

$$
\begin{gathered}
\left(K-K_{\mathrm{UKIDSS}}\right)_{\mathrm{Vega}}=-0.09+0.27 \cdot(H-K)_{\mathrm{UKIDSS}} \\
\text { for } K<17.0 \mathrm{mag} .
\end{gathered}
$$

The right panel of Figure 10 illustrates the external photometric accuracy after this simple color-term correction. The photometric comparison between UKIDSS and WIRCam data is affected by a statistical uncertainty of 0.05 mag down to about $K_{s}=17$ Vega mag, which corresponds to $\sim 18.82 \mathrm{AB}$ mag. 

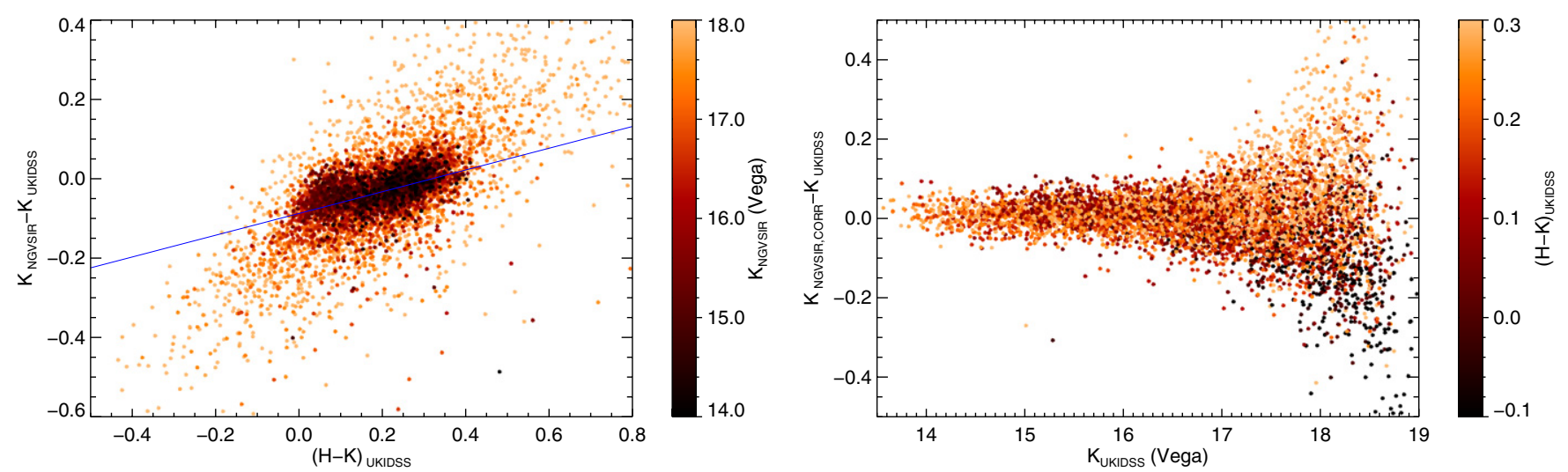

Figure 10. Left: Color term resulting from the differences between the UKIDSS and WIRCam $K_{s}$ filters. The shown stellar sample is the same as in the right panel of Figure 9. WIRCam $K_{s}$ aperture photometry (in Vega magnitudes) is used for color coding, and the best linear fit is indicated by the solid line. Right: Same as the right panel of Figure 9, but after applying the color-term correction to the $K_{S}$ NGVS-IR data. The symbol color is parameterized according to the $(H-K)_{\text {UKIDSS }}$ color, as indicated by the color bar next to the panel.

(A color version of this figure is available in the online journal.)

Table 2

Average Zero Points of Photometric Nights

\begin{tabular}{|c|c|c|c|}
\hline Program & MJD & $N_{\text {phot }}{ }^{\mathrm{a}}$ & $\overline{z p}_{K}$ \\
\hline \multirow[t]{6}{*}{$09 \mathrm{BC} 26$} & 55187 & 71 & $23.16 \pm 0.01$ \\
\hline & 55190 & 138 & $23.14 \pm 0.02$ \\
\hline & 55191 & 71 & $23.14 \pm 0.02$ \\
\hline & 55192 & 55 & $23.12 \pm 0.01$ \\
\hline & 55194 & 123 & $23.13 \pm 0.02$ \\
\hline & 55195 & 159 & $23.14 \pm 0.01$ \\
\hline 09BF22 & 55191 & 71 & $23.12 \pm 0.01$ \\
\hline \multirow[t]{14}{*}{$10 \mathrm{AC} 10$} & 55280 & 128 & $23.14 \pm 0.02$ \\
\hline & 55283 & 44 & $23.11 \pm 0.02$ \\
\hline & 55287 & 129 & $23.14 \pm 0.01$ \\
\hline & 55288 & 60 & $23.10 \pm 0.02$ \\
\hline & 55290 & 3 & $23.13 \pm 0.01$ \\
\hline & 55291 & 33 & $23.13 \pm 0.02$ \\
\hline & 55308 & 59 & $23.12 \pm 0.02$ \\
\hline & 55310 & 256 & $23.15 \pm 0.03$ \\
\hline & 55311 & 78 & $23.15 \pm 0.02$ \\
\hline & 55342 & 84 & $23.14 \pm 0.02$ \\
\hline & 55343 & 111 & $23.15 \pm 0.01$ \\
\hline & 55374 & 56 & $23.11 \pm 0.03$ \\
\hline & 55379 & 37 & $23.13 \pm 0.03$ \\
\hline & 55380 & 70 & $23.11 \pm 0.02$ \\
\hline \multirow[t]{12}{*}{ 10AF03 } & 55287 & 4 & $23.15 \pm 0.01$ \\
\hline & 55288 & 144 & $23.11 \pm 0.01$ \\
\hline & 55290 & 38 & $23.14 \pm 0.02$ \\
\hline & 55291 & 132 & $23.11 \pm 0.02$ \\
\hline & 55316 & 143 & $23.12 \pm 0.02$ \\
\hline & 55317 & 32 & $23.13 \pm 0.02$ \\
\hline & 55320 & 36 & $23.12 \pm 0.02$ \\
\hline & 55322 & 114 & $23.11 \pm 0.02$ \\
\hline & 55341 & 54 & $23.15 \pm 0.01$ \\
\hline & 55343 & 46 & $23.15 \pm 0.02$ \\
\hline & 55382 & 57 & $23.12 \pm 0.02$ \\
\hline & 55383 & 41 & $23.14 \pm 0.02$ \\
\hline
\end{tabular}

Note. ${ }^{a}$ Number of photometric frames.

\section{COMPLETENESS ESTIMATES}

The photometric completeness of point sources in the final image stacks was computed by adding artificial stars with a range of magnitudes at randomly selected positions into the image. We use a set of IDL and Python scripts to generate a list with the positions and magnitudes of artificial stars, avoiding areas with the presence of saturated stars and very extended galaxies, e.g., the central regions of M87.

The first step consists of generating the positions of artificial stars. We run SExtractor on the final images and generate the segmentation maps. We use these maps to avoid adding stars on top of a galaxy or other high-density regions. Then the image is divided into a two-dimensional grid of 200 pixel wide bins, and we randomly generate 10 positions in each bin. For each of these positions, we check that there is no collision with other sources (real or artificial) in a radius of 16 pixels, thereby avoiding artificial crowding effects. The final list consists of about 100,000 sources for each final quadrant image.

In the second step, we generate the magnitudes of the artificial stars. First we run PSFex on the final images and compute a position-dependent PSF model. We use this to properly add a pointlike source in different positions on the image. Then we generate a homogeneous distribution of magnitudes in the range $18.0<K_{\text {Vega }}<22.0$ and assign them to the stars generated in the previous step ( $\left.K_{\text {input }}\right)$.

The third step adds the artificial stars to the image at the corresponding positions, with the generated magnitudes using the appropriate PSF model. We use a highly efficient multithreaded Python script for reading the list of coordinates and building the pointlike sources according to the PSF model computed with PSFex using all available CPU cores.

We ran 200 realizations of this procedure for each NGVS-IR quadrant, ending up with $2 \times 10^{7}$ artificial objects per quadrant. We ran SExtractor with the same parameters used for the original stacks (see Section 4) and cross-matched the positions of the detected sources with the artificial stars generated in step 1, hereafter called recovered stars. The top panel of Figure 11 shows the difference between the magnitude of artificial stars in tile $+0+0$ as measured with SExtractor $\left(K_{\text {output }}\right)$ and assigned in the mock catalog $\left(K_{\text {input }}\right)$. This figure illustrates the internal photometric errors of the NGVS-IR survey and demonstrates the excellent quality of the stacked images. In order to estimate the completeness, we adopt a magnitude bin of $0.1 \mathrm{mag}$ and compute the ratio between the number of recovered stars and the number of artificial stars as a function of magnitude and position. The bottom panel of Figure 11 shows the completeness fraction as a function of the input $\mathrm{AB}$ magnitude for each of the 


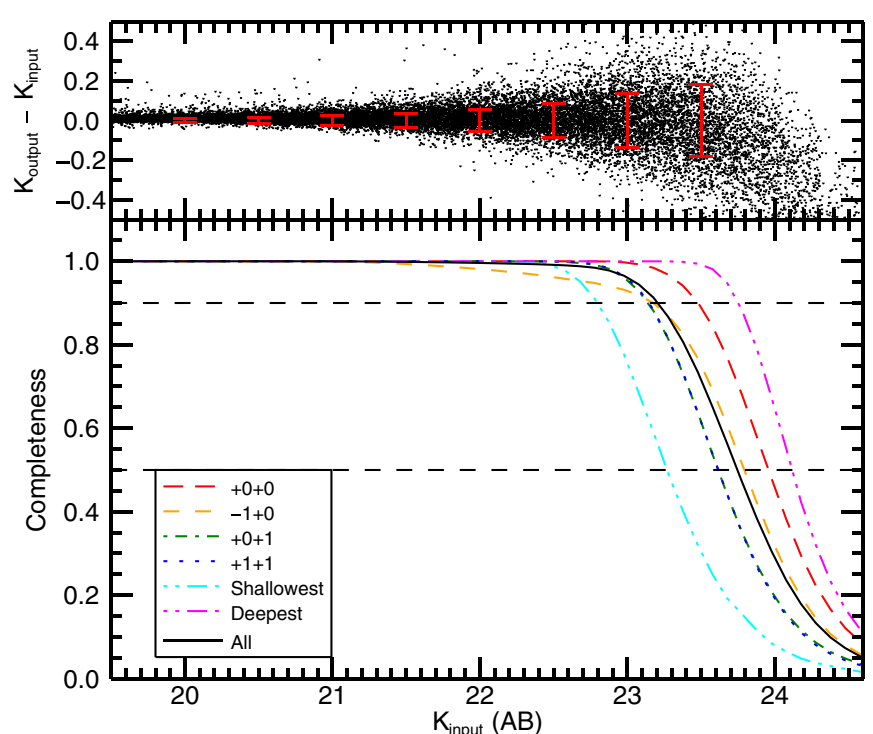

Figure 11. Top: Internal photometric errors for tile $+0+0$ of NGVS-IR. The errors were computed by adding artificial stars to the stacked image and then running SExtractor for the photometry. Bottom: Photometric completeness curves for the four quadrants of the NGVS-IR pilot program region, plus the deepest and shallowest regions in the survey. The horizontal dashed lines correspond to $90 \%$ and $50 \%$ completeness.

(A color version of this figure is available in the online journal.)

four quadrants, the entire survey, and the deepest and shallowest regions in the pilot field. The $90 \%$ completeness magnitudes for point-source detections and the corresponding $5 \sigma$ limiting magnitudes together with a conservative surface brightness limit estimate for the NGVS-IR quadrants are summarized in Table 3.

Figure 12 illustrates the variations of all the completeness magnitudes across the four quadrants. The main reason for the fluctuations visible across the field stems from the photometric quality, i.e., FWHM distribution (see Figure 6), in each individual WIRCam tile contributing to the final quadrant stack.

\section{DISCUSSION}

\subsection{Color-Color Diagrams}

Adding more filters and successively wider SED coverage to the investigation of astronomical objects adds more diagnostic power to the analysis and delivers ultimately more robust and astrophysically meaningful results (e.g., Park \& Choi 2005; Puzia et al. 2007). Color-color planes are efficient tools for the classification of sources in large-scale imaging surveys (e.g., Daddi et al. 2004; Faber et al. 2007). By combining near-UV and

Table 3

Completeness Magnitude of the NGVS-IR Stacks

\begin{tabular}{lccc}
\hline \hline Tile & $K_{\mathrm{AB}, 90 \%}$ & $K_{\mathrm{AB}, 5 \sigma}$ & $\mu_{K_{s}, \mathrm{AB}, 5 \sigma}$ \\
\hline$+0+0$ & 23.70 & 24.64 & 25.11 \\
$-1+0$ & 23.51 & 24.61 & 25.08 \\
$+0+1$ & 23.32 & 24.16 & 24.63 \\
$+1+1$ & 23.34 & 24.23 & 24.70 \\
\hline
\end{tabular}

Note. The $5 \sigma$ limiting magnitude is measured inside a circular aperture with 0.7 radius and marks the limiting surface brightness estimate. optical data from NGVS and near-IR photometry from NGVSIR, the advantage of spanning the entire spectral range of stellar emission from the atmospheric UV cutoff at $\sim 3200 \AA$ to the near-IR at $\sim 2.5 \mu \mathrm{m}$ becomes clear when one is confronted with the vastly improved system throughputs and SED coverage of the combined NGVS + NGVS-IR filter set, as illustrated in Figure 13.

To construct a first NGVS + NGVS-IR source catalog, we cross-matched the NGVS-IR catalog positions with those of the NGVS $u^{*}$ griz catalog, which contains approximately half a million objects. The distribution of angular separations between $K_{s^{-}}$and $i$-band coordinates peaks at 0.09 , and $95 \%$ of the detected sources (of small angular size) have a separation smaller than 0 .'3. This small bias is due to the marginally resolved nature of background galaxies, which constitute a large fraction of the matched catalog and introduce a random offset in the matching accuracy that appears as a systematic in one radial dimension. Future dedicated versions of these matched catalogs will be built with object-dependent procedures that allow for self-consistent optical and near-IR measurements, in particular for extended sources. However, independent measurements and subsequent matching are fully sufficient for the qualitative purposes of this paper.

Historically, the optical/near-IR color-color plane that is most widely used in conjunction with large extragalactic surveys is the $B z K$ diagram, which combines $B-z$ and $z-K$ color indexes or equivalents thereof. The diagram was highlighted by Daddi et al. (2004) as a means of identifying both passively evolving and star-forming galaxies located at redshifts larger than 1.4 or so. It was published later for a variety of deep fields, confirming the original segregation between low- and high-redshift sources (e.g., Lane et al. 2007; McCracken et al. 2010, 2012; Bielby et al. 2012). The $B z K$ technique is now frequently used for studies of the clustering properties, intrinsic morphologies, stellar populations, and X-ray luminosities of $B z K$-selected galaxies (e.g., Lin et al. 2012; Yuma et al. 2011, 2012; Ly et al. 2012; Rangel et al. 2013). Ultradeep follow-up spectroscopy campaigns confirm the efficient selection of these types of high-redshift, star-forming galaxies, suffering $\sim 10 \%$ contamination by other sources (Kurk et al. 2013).

\subsection{The NGVS + NGVS-IR Analog of BzK: The $g z K_{s}$ Color-Color Plane}

In terms of SED coverage of the involved filters, the closest analog to the $B z K$ diagram that can be constructed from our combined NGVS + NGVS-IR data is the $g z K_{s}$ diagram, which is shown in the top panel of Figure 14, which illustrates our pilotfield sample gray-scale-coded by the Gaussian-propagated total error of each contributing filter (see also Figure 13 for a comparison of the corresponding $g z K_{s}$ versus $B z K$ system throughput curves). The mean foreground extinction toward M87 is $A(g) \simeq 0.076, A(z) \simeq 0.029$, and $A\left(K_{s}\right) \simeq 0.007 \mathrm{mag}$ and was taken from Schlafly \& Finkbeiner (2011). ${ }^{32}$ The following analysis includes the corresponding extinction correction terms. We observe various characteristic object overdensities and sequences in the $g_{z} K_{s}$ plane, such as the narrow sequence of foreground Galactic stars and other less constrained object distributions, including actively star-forming and passively evolving galaxies at various redshifts, that were discussed in previous works (e.g., Bielby et al. 2012; Merson et al. 2013). We use

\footnotetext{
32 See Appendix B for a discussion of the small color dependence of the extinction coefficients.
} 

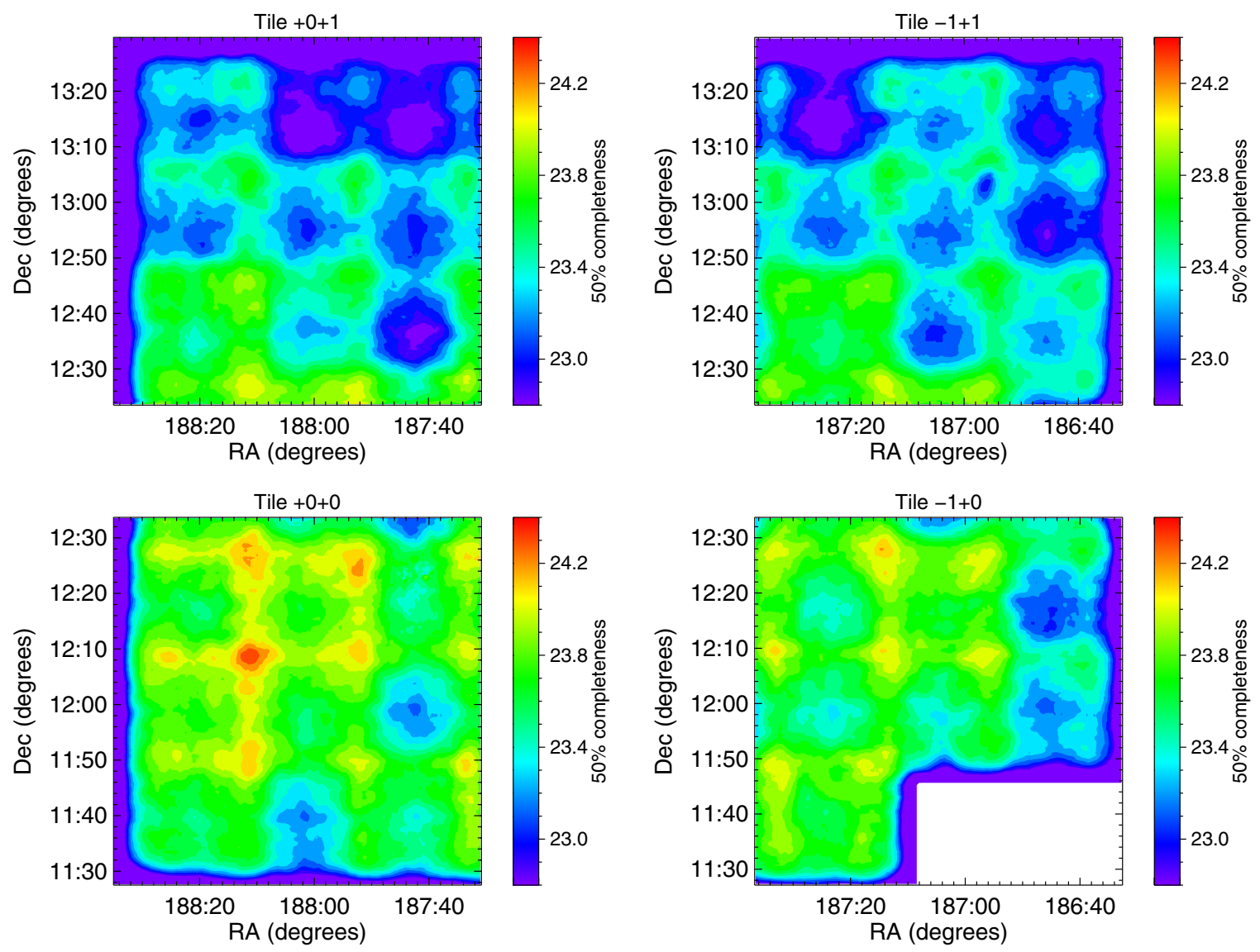

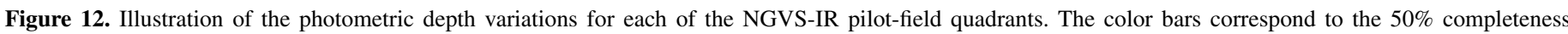
magnitude of pointlike source detections. The structures in each field are mainly due to the seeing distribution of the corresponding tiles and their overlap regions.

(A color version of this figure is available in the online journal.)

Equations (1)-(8) provided by Bielby et al. (2012) to transform the $B z K$ object selections into our $g z K_{s}$ color-color plane. In particular, we use the median color $u-g=-0.194$ mag of our entire sample and obtain for the selection of star-forming galaxies at $z \gtrsim 1.4$ the following relation:

$$
\left(z-K_{s}\right)_{0}>1.233 \times(g-z)_{0}-0.017
$$

(Figure 14, solid line). To select passively evolving galaxies at $z \gtrsim 1.4$, we obtain

$$
\left(z-K_{s}\right)_{0}<1.233 \times(g-z)_{0}-0.017 \cap\left(z-K_{s}\right)_{0}>2.5
$$

(Figure 14). To select foreground stars and separate them from galaxies, our transformations yield (dotted magenta line in Figure 14)

$$
\left(z-K_{s}\right)_{0}<0.37 \times(g-z)_{0}+0.474 .
$$

The corresponding relations shown in the top panel of Figure 14 illustrate the quality of separating the classically defined $B z K$ galaxies at $z>1.4$ from the general locus of more nearby galaxies and the stellar sequence. However, it is quite evident that even with the NGVS + NGVS-IR high-quality data, a clear separation of stellar foreground from the background galaxy population is not entirely possible based on the $g z K_{s}$ color-color diagram alone.

Overall, the $g z K_{s}$ color-color plane is the tool of choice for the study of the redshifted universe beyond Virgo because of the superior photometric depth of the $g$ filter compared with $u^{*}$-band observations. However, unlike any other deep optical/near-IR survey, NGVS + NGVS-IR also contains a nearby structure: Virgo itself. The most striking difference between our color-color diagrams and those of other surveys is not due to Virgo galaxies - these occur in negligible numbers in any given part of the diagram - but to GCs that are concentrated around the massive elliptical galaxy NGC 4486 (M87) and other giant elliptical galaxies located in the central regions of Virgo (see Figure 3). To demonstrate the locus of $g z K_{s}$ colors of Virgo GCs, we highlight radial-velocity-confirmed GCs in Figure 14 (red circles; E. W. Peng et al. 2014, in preparation). This GC locus overlaps with the colors of stars at the blue end and is heavily contaminated by background galaxies toward redder colors in the $g z K_{s}$ diagram.

\subsection{The uiK $K_{s}$ Color-Color Plane}

For a photometry-based selection of GCs and other compact stellar systems, such as UCDs and dwarf galaxies, we show in the following that the most powerful color combination is given by the $u i K_{s}$ diagram, which combines high-quality near-UV, optical, and near-IR photometry from NGVS and NGVS-IR. The bottom panel of Figure 14 shows the $u i K_{s}$ color-color diagram, in which the data are dereddened with the values extracted from the Schlafly \& Finkbeiner (2011) maps, using $A(u) \simeq 0.097, A(i) \simeq 0.039$, and $A\left(K_{s}\right) \simeq 0.007 \mathrm{mag}$. The typical structures seen in the $g z K_{s}$ diagram (Figure 14, top) that were classified in previous deep-field surveys appear much more 

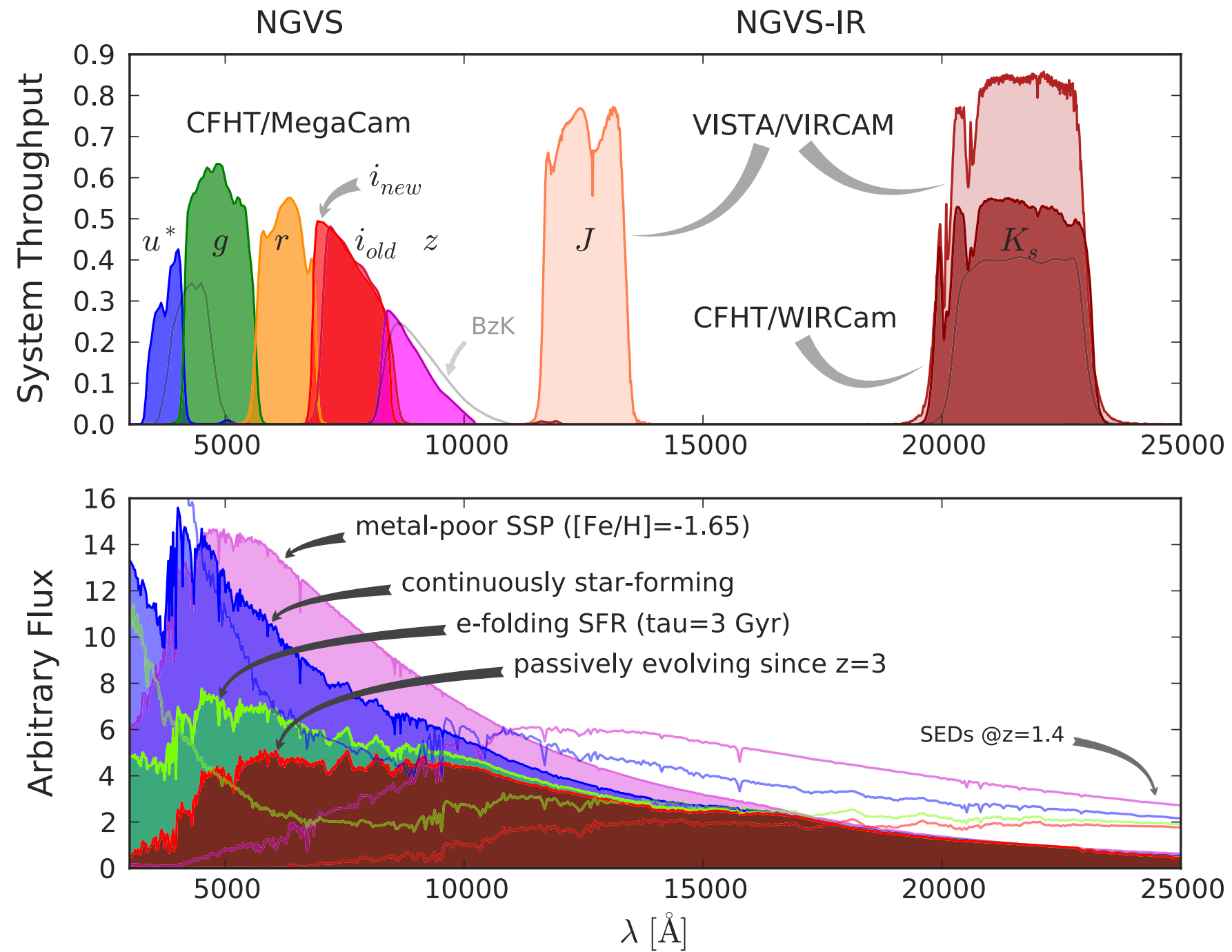

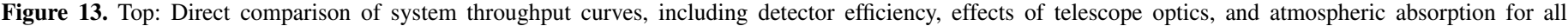

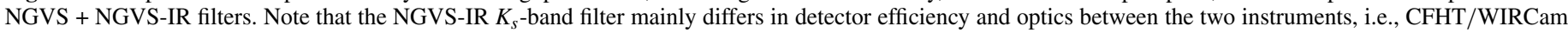

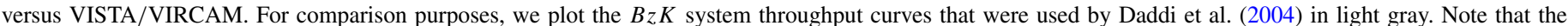

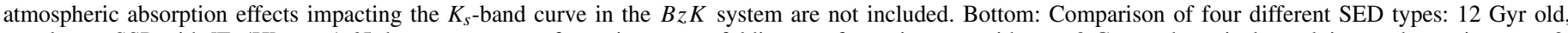

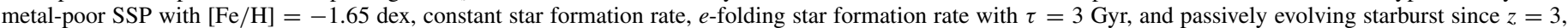

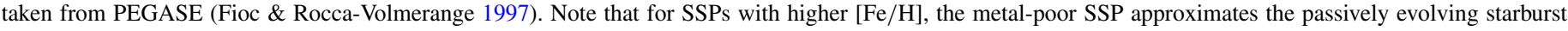

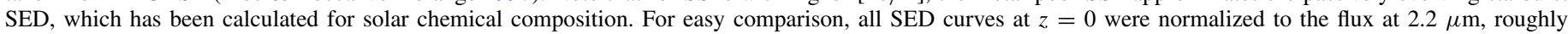
corresponding to the effective wavelength of the $K_{s}$ band. The open SED curves correspond to the same four SED types as they would appear at $z=1.4$.

(A color version of this figure is available in the online journal.)

prominent and better defined in the $u i K_{s}$ color-color plane. The stellar sequence seen in the $u i K_{s}$ diagram remains very clearly identified in this new plane and appears more separated from the main cloud of galaxies. This will be particularly useful for the analysis of stellar age and metallicity distribution functions in the Virgo overdensity described in Ferrarese et al. (2012). Several new, narrowly defined features in the $u i K_{s}$ plane become visible at intermediate colors, which we attempt to classify qualitatively in the following. Most importantly, however, we note that the contamination of the GC locus is very significantly reduced with the use of the $u i K_{s}$ filter combination.

\subsubsection{An Efficient Tool for Star Cluster Selection}

In order to better understand the various features in the $u i K_{s}$ plane, we overplot in Figure 15 predictions of simple stellar population (SSP) model calculations based on a customized version of the population synthesis code PEGASE (Fioc \& Rocca-Volmerange 1997) that includes the exactly matched throughput functions for all NGVS + NGVS-IR filters. While in the $g z K_{s}$ plot these SSP models coincide with the overlap region between stars and galaxies, in the $u i K_{s}$ plane they fall right on top of a sharply defined sequence, which we identify as GCs. We note that the metallicity and age coverage of the SSP model predictions, i.e., $Z=0.0004,0.001,0.004$, and 0.02 and $t=8-13$ Gyr (each running from bluer to redder colors), agree fairly well with the GC candidate locus, in particular in the $u i K_{s}$ plane, and is consistent with what is expected from previous photometric and spectroscopic studies of stellar populations in typical Virgo GCs located in the vicinity of M87 (e.g., Hanes \& Brodie 1986; Cohen et al. 1998; Hanes et al. 2001; KisslerPatig et al. 2002; Jordán et al. 2002; Tamura et al. 2006b; Peng et al. 2009; Yoon et al. 2011; Forte et al. 2013). We verify the fidelity of the GC selection with objects that have the systemic radial velocity of the Virgo Cluster using spectroscopy available in the literature (Hanes et al. 2001; Strader et al. 2011) and recently obtained with the multiobject, moderate-dispersion 

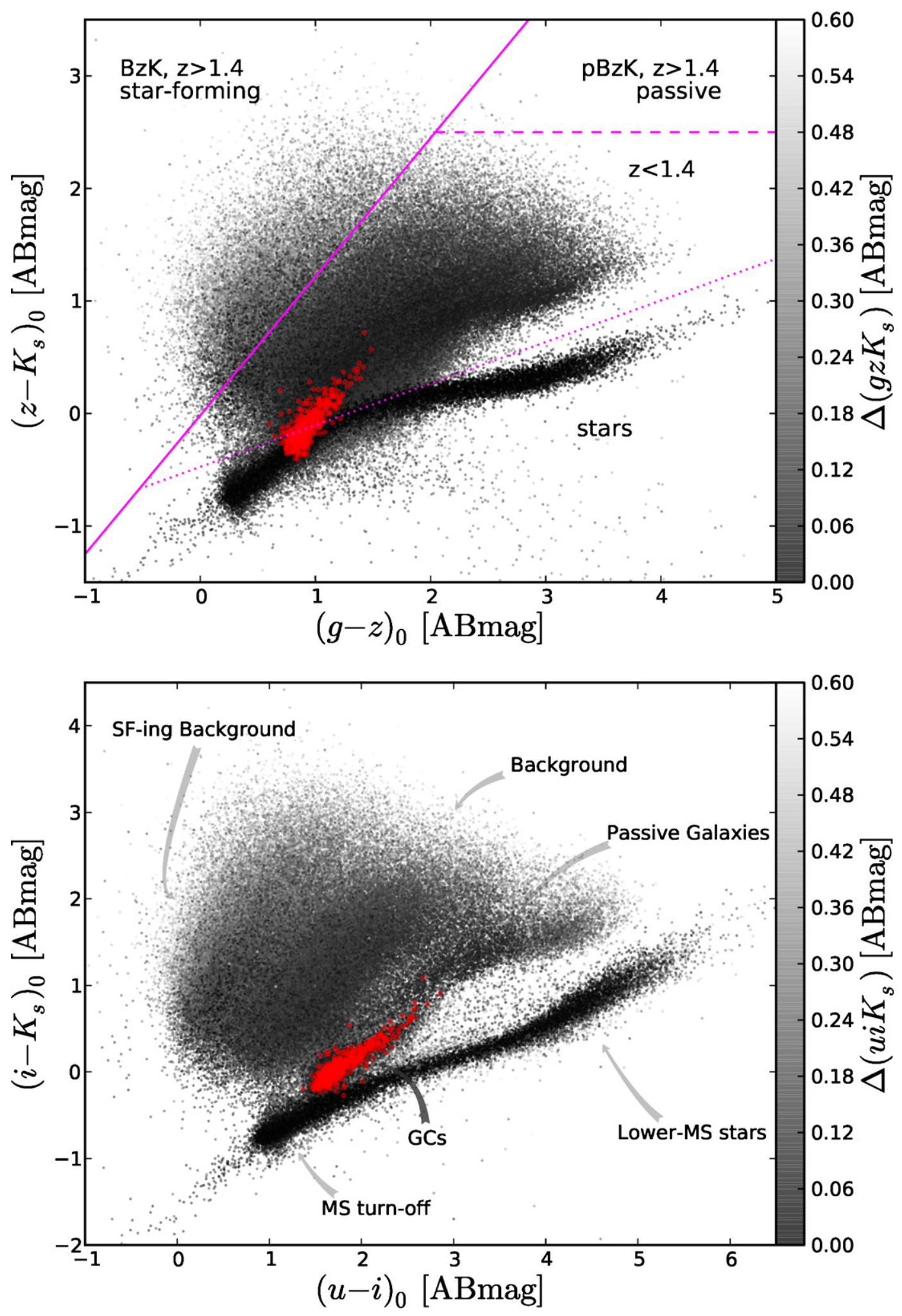

Figure 14. Illustration of the $g z K_{s}$ (top) and $u i K$ (bottom) color-color diagrams for all the objects in the NGVS pilot field (gray dots). The symbol shading is parameterized by the total photometric error of each filter contributing to each color plane. All magnitudes are in the AB system and include the reddening corrections from Schlafly \& Finkbeiner (2011). The red circles mark spectroscopically confirmed globular clusters with the systemic velocity of Virgo Cluster galaxies (see text for details). The solid magenta line shows the criteria defined by Daddi et al. (2004) to isolate $z>1.4$ star-forming galaxies, the dashed line to separate $z>1.4$ passively evolving galaxies from nearby systems, and the dotted line to separate foreground stars from galaxies. The corresponding $B z K$ areas are labeled accordingly in the top panel. We indicate in the bottom panel characteristic object sequences (i.e., stars, GCs, and passively evolving galaxies) and other overdensities (locations of normal and star-forming background galaxies) that are discussed in Section 6.3.

(A color version of this figure is available in the online journal.) 

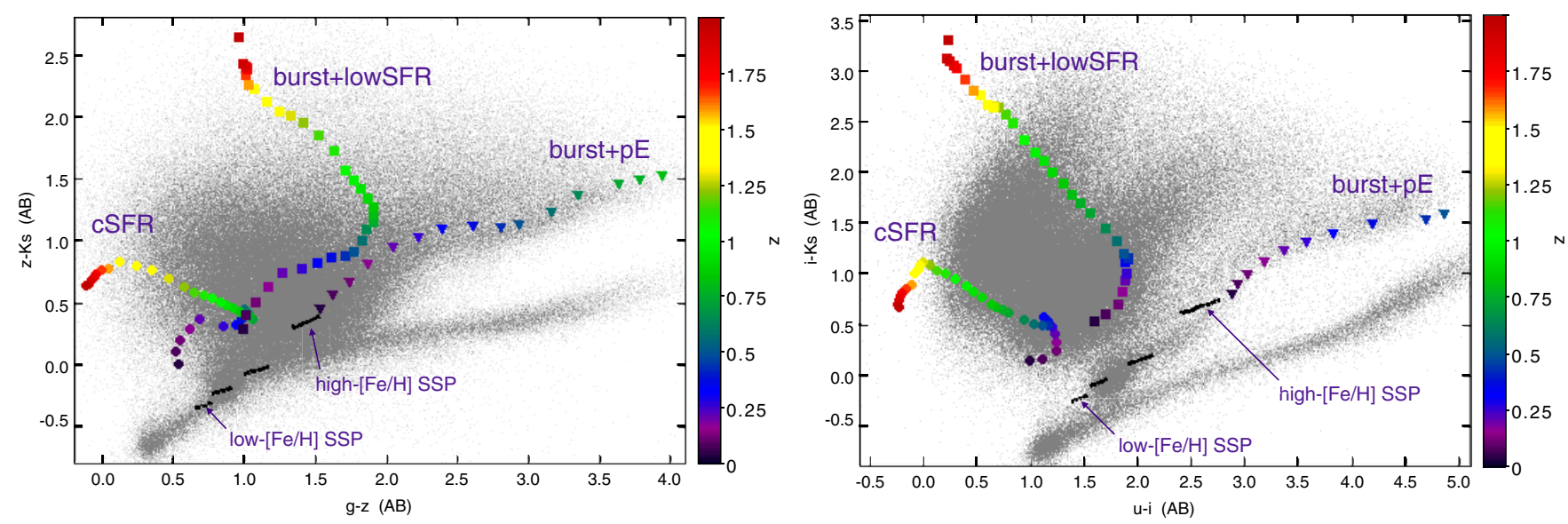

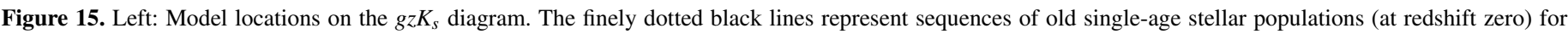

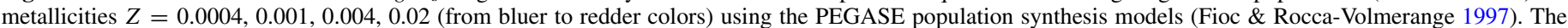

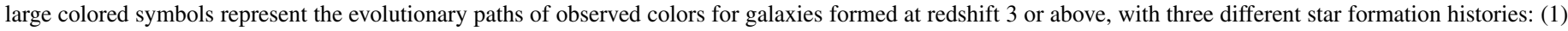

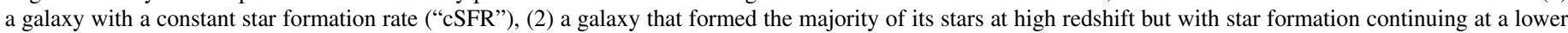

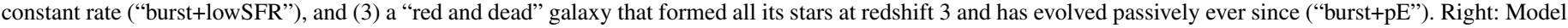
locations in the $u i K_{s}$ diagram, with symbols as in the left panel.

(A color version of this figure is available in the online journal.)

spectrograph Hectospec at the Multi-Mirror Telescope (MMT; E. W. Peng et al. 2014, in preparation). In Figure 14, we overplot these radial-velocity-confirmed Virgo GCs in the $g z K_{s}$ and $u i K_{s}$ color-color diagrams as red circles. At this point, we defer a more quantitative analysis of the Virgo GC stellar populations to a future paper and continue with the investigation of the other sequences, keeping in mind the $u i K_{s}$-based photometric GC selection.

\subsubsection{Redshift Evolution of Galaxies in the uiK $K_{\text {S }}$ Plane}

In addition to the SSP model color predictions, we plot in Figure 15 the redshift evolution in the $g z K_{s}$ and $u i K_{s}$ color-color space of three prototypical SEDs of galaxies born at $z=3$ with different types of star formation history. These tracks were computed using the NGVS + NGVS-IR instrument throughput curves (see Figure 13) with a customized version of the population synthesis code PEGASE (Fioc \& RoccaVolmerange 1997), but other population synthesis codes show very similar trends (see Dahlen et al. 2013). In order of numerically increasing colors, the tracks correspond to (1) a continuously star-forming galaxy, (2) a galaxy that formed the majority of its stellar content at high redshift with star formation that continues at a lower constant rate, and (3) a passively evolving galaxy that formed all its stellar content at $z=3$.

We note that, in contrast to the $g z K_{s}$ plane, galaxies with any level of star formation are expected to be fairly distinct in their $u i K_{s}$ color properties from GCs and foreground stars from $z=0$ out to redshifts $z \simeq 1.5$ and higher. We find that the hook in the redshift evolution of starbursting galaxies at $z \approx 0.5-1$ in the $g z K_{s}$ color-color plane is responsible for much of the contamination at colors $g-z \simeq 1.0$ and $z-K_{s} \simeq 0-0.5 \mathrm{mag}$, which is the mean locus of metal-poor and intermediate-metallicity GCs. Such contamination is entirely avoided in the $u i K_{s}$ plane, as a result of the mostly blueward evolution of the $u-i$ color when redshifting the steeply increasing near-UV part of star-forming galaxy SEDs. The redshift evolution of the corresponding passively evolving SEDs overlaps with the local galaxy background of Virgo, and its diagnostic power is only limited by the depth of our $u^{*}$-band photometry. The shallower magnitude limit of the $u^{*}$-band data is apparent when the blue edge of the galaxy distribution in each diagram of Figure 15 is compared. Although the redshift distribution of the background galaxies in the $u i K_{s}$ plane cuts off at lower values as compared with the $g z K_{s}$ plane, especially along the sequence of passively evolving galaxies (the cutoff is near $z \approx 0.5$ instead of $z \approx 1$ ), the $u i K_{s}$ plane provides overall a clearly linear and powerful selection diagnostic for star-forming galaxies in the redshift range $z \simeq 0.5-1.5$. Because of this property and the photometric depth of the NGVS + NGVS-IR data, we are in the position of searching for galaxy clusters at redshifts $z \gtrsim 1.0$, which will be described in detail in future papers.

\subsubsection{Diagnostic Power of the uiK $K_{s}$ Plane}

The reason for the superior GC-star and GC-galaxy separation power of the $u i K_{s}$ plane lies in the combination of the near-UV, optical, and near-IR color information, compared with the classic optical/near-IR $g z K_{s}$ plot (see Figure 14). While the optical/near-IR $i-K_{s}$ color probes the effective temperature of the red giant branch (RGB) stellar population, the nearUV/optical $u-i$ color probes the stellar fluxes blueward and redward of the $4000 \AA$ break and is, therefore, sensitive to hot stellar evolutionary phases. This leads to a very efficient GC-galaxy separation due to the sensitivity of the $u$ band to hot stellar components in star-forming galaxies, combined with the redshift evolution of galaxy SEDs (see Figures 13 and 15). The efficient GC-star separation in the $u i K_{s}$ diagram is due to the fact that at a given $i-K_{s}$ color (i.e., effective red giant branch temperature), the $u$ band picks up the additional hot stellar component flux generated by horizontal-branch stars, making GCs appear bluer in $u-i$ color.

It is worth pointing out that attempts at photometrically separating extragalactic GCs from foreground stars and background galaxies exist in the recent literature, however, based on a combination of purely optical/near-IR (Puzia et al. 2002) or purely near-UV/optical colors (Kim et al. 2013). Neither of these color-color planes can separate foreground stars, GCs, and background galaxies. The only earlier study that combined near-UV, optical, and near-IR photometry of relatively small GC samples was attempting to study the age distribution 

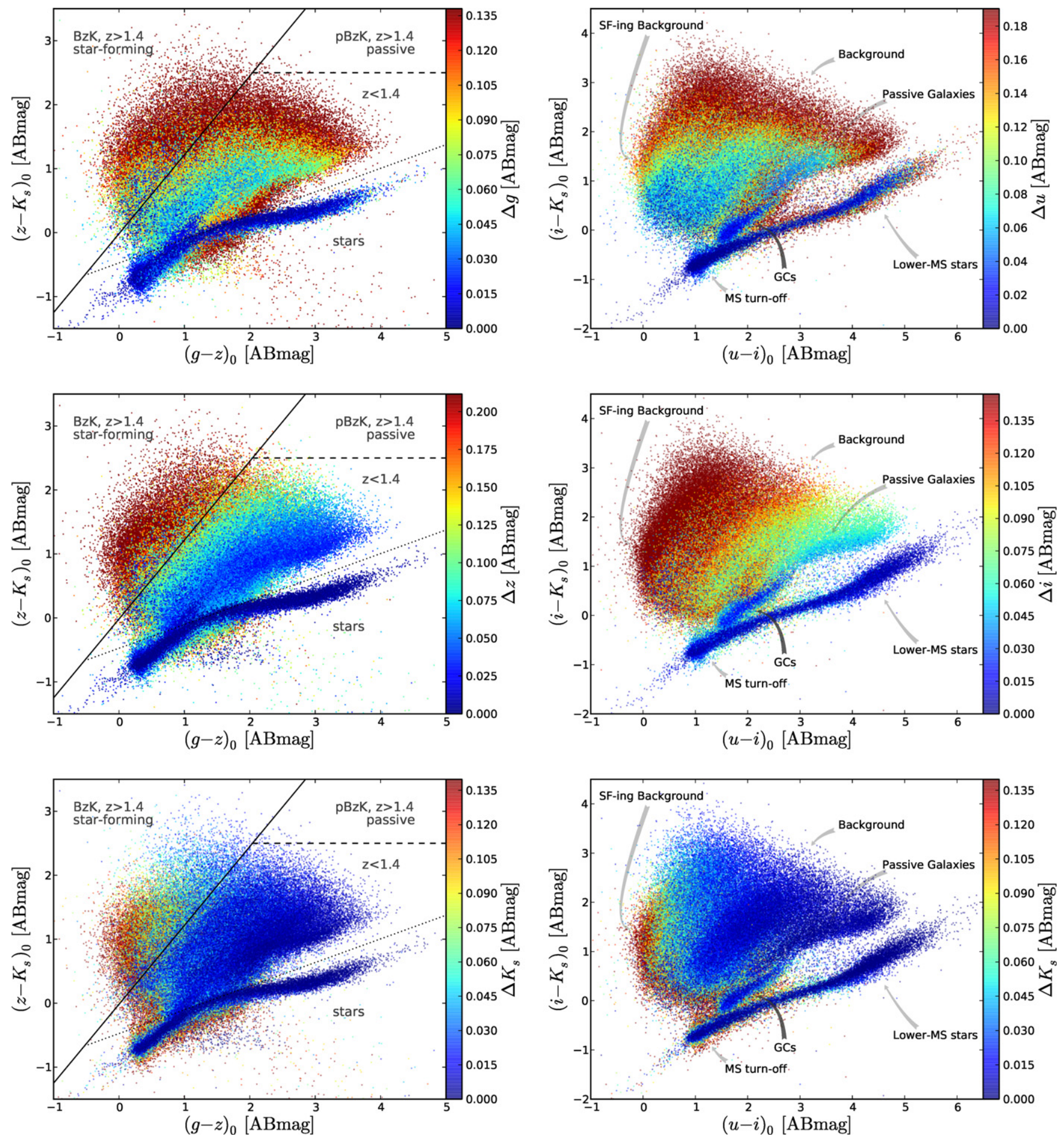

Figure 16. Left: Illustration of the $g z K_{s}$ color-color diagram, which is the best approximation to the classic $B z K$ diagram using the NGVS + NGVS-IR filter set. The plots show the entire data set in the Virgo pilot field. The lines mark the separations between star-forming galaxies at $z>1.4$, passively evolving galaxies at $z>1.4$, galaxies at $z<1.4$, and foreground stars as defined by Daddi et al. (2004). Color bars indicate the color parameterization of the symbols in each panel by one of the constituent $g z K_{s}$ filters, i.e., in the $g$-band (top), $z$-band (middle), and the $K_{s}$ filter (bottom). Right: The corresponding $u i K_{s}$ color-color diagrams of objects in the NGVS pilot-field region. The color bars indicate the photometric error parameterization in the $u$ band (top), $i$ band (middle), and $K_{s}$ band (bottom).

(A color version of this figure is available in the online journal.)

function of GCs in giant elliptical galaxies (Hempel \& KisslerPatig 2004) and was based on much poorer photometric quality. To our knowledge, the $u i K_{s}$ diagnostic plane presented in Figure 14 is the first such plot based on high-quality nearUV/optical/near-IR photometry that allows for efficient GC-star and GC-galaxy separation.
In order to assess the required photometric quality for robust GC-star and GC-galaxy separation, we plot in Figure 16 the $g z K_{s}$ and $u i K_{s}$ color-color planes and parameterize the symbol colors by the photometric uncertainty in each contributing filter. It is immediately clear that the photometric depth, i.e., photometric error distribution function in each filter, influences 
in a nonlinear, and sometimes quite dramatic, way the selection of $B z K$ galaxies as defined by Daddi et al. (2004). The corresponding relations are shown in the left panels (as in Figure 14) and illustrate the quality of selecting the classically defined galaxy samples as a function of photometric depth. It is also quite evident that even with the NGVS + NGVS-IR highquality data, a clear separation of stellar foreground from the background galaxy population is not entirely possible based on the $g z K_{s}$ color-color diagram alone; in particular, Virgo GCs cannot be robustly identified.

The right panels of Figure 16 demonstrate the high potential for studies of stellar populations in the nearby universe. We find that data sets with a photometric accuracy of $\Delta u \lesssim 0.05$, $\Delta i \lesssim 0.05$, and $\Delta K_{s} \lesssim 0.05 \mathrm{mag}$ (corresponding to $u \cong 24.4$, $i \simeq 23.4$, and $K_{s} \simeq 22.0 \mathrm{AB}$ mag in our data set) will have the potential to select very clean samples of extragalactic GCs. We will discuss and quantify the exact contamination fractions of such samples in future papers.

With the superior spatial resolution of our $K_{s}$-band data (median seeing 0.'54; see Section 3.6 and Figure 6), we search for additional diagnostic tools related to the morphology of objects in our survey field. For this, we devise a simple magnitude difference between a 3 " diameter aperture magnitude and a PSF magnitude. The PSF magnitude produces integrated colors for stars very similar to the total integrated colors for GCs, and a color roughly representative of the central colors for galaxies. The results for all our objects with $K_{s}$-band photometry are shown in Figure 17 as a color parameterization of the $u i K_{s}$ plane according to the morphological measure of their radially symmetric compactness. Intriguing correlations between this simple morphology parameter and $u i K_{s}$ colors appear, which will be studied in subsequent papers of this series. We note here that the figure clearly shows that GCs are marginally resolved in the WIRCam $K_{s}$ images: their observed shapes are slightly less concentrated than those of stars. The accuracy of measuring relative GC sizes is sufficient to even make out the classic size difference between blue and red GCs (e.g., Webb et al. 2012 and references therein). Attempts to identify GCs solely on the basis of their morphology, however, produce samples whose colors reveal a high level of contamination by stars and remote galaxies. Our final classification algorithm (in preparation) will therefore be based on both colors and morphology.

\section{SUMMARY}

We have described the NGVS-IR survey, its motivation, observational strategy, and science goals. We discuss in detail the data reduction procedures and present $K_{s}$-band imaging data of the central $4 \mathrm{deg}^{2}$ of the Virgo galaxy cluster observed with WIRCam, mounted on the Canada-France-Hawaii Telescope. These NGVS-IR images have a median seeing of 0.54 and a $90 \%$ completeness magnitude of better than $K_{s}=23.3 \mathrm{AB}$ mag, thus superseding in all aspects the 2MASS and UKIDSS mosaics of the same region.

Using the full near-UV to near-IR SED coverage of our NGVS + NGVS-IR data, we investigated the $u i K_{s}$ color-color plane as a diagnostic tool to identify and select characteristic object classes. We also studied the $g z K_{s}$ color-color plane as the closest equivalent to the $B z K$ plot, from which star-forming galaxies at redshift $z>1.4$ are typically selected. With the described $u i K_{s}$ diagnostics, we can identify several distinct and isolated groups of objects in the $u i K_{s}$ diagram that correspond to (1) star-forming galaxies out to redshifts $\sim 1.5$ and higher, (2) a mixed distribution of galaxies with a variety of (nonnegligible) levels of ongoing star formation, (3) a redshift sequence of passively evolving old galaxies, (4) a relatively smooth sequence of Virgo GCs and UCDs, and (5) a very distinct sequence of foreground Milky Way stars.

According to the photometric error distributions of our NGVS + NGVS-IR data in the $u i K_{s}$ color-color diagram (see Figure 16, right), we can isolate the Virgo GC population, virtually free of contamination from foreground stars and background galaxies, if we restrict our selection to objects with photometric errors $\Delta u \lesssim 0.05, \Delta i \lesssim 0.05$ and $\Delta K_{s} \lesssim 0.05 \mathrm{mag}$. Independent of the photometric quality of the data set, such a selection would be fundamentally impossible from the $g z K_{s}$ diagram. The $u i K_{s}$ diagram is, to our knowledge, the most efficient and least biased extragalactic GC selection technique that relies purely on photometric colors and is likely to prove extremely efficient in the study of distant star cluster systems with the combined near-UV/optical/near-IR instrumentation that will be available on the upcoming generation of survey telescopes, such as the Large Synoptic Survey Telescope and the Euclid spacecraft, as well as future facilities, such as the European Extremely Large Telescope and James Webb Space Telescope.

This research was supported by CONICYT through Gemini-CONICYT Project 32100022, FONDECYT Regular Project 1121005, FONDECYT Postdoctoral Fellowship Project 3130750, the FONDAP Center for Astrophysics (15010003), the BASAL Center for Astrophysics and Associated Technologies (PFB-06), and the French Agence Nationale de la Recherche (ANR) Grant Programme Blanc VIRAGE (ANR10BANC-0506-01). R.P.M. and A.L. acknowledge support from the Scientific Council of Strasbourg University. A.L. is grateful for the hospitality and financial support during her stay at the Instituto de Astrofísica of Pontificia Universidad Católica de Chile, which was funded by the UMI International Academic Exchange Fund. E.W.P. acknowledges support from the National Natural Science Foundation of China (grant 11173003) and from the Laboratoire International Associé "ORIGINS." C.L. acknowledges support from the National Natural Science Foundation of China (grant 11203017).

Based on observations obtained with WIRCam, a joint project of CFHT, Taiwan, Korea, Canada, France, at the Canada-France-Hawaii Telescope (CFHT), which is operated by the National Research Council (NRC) of Canada, the Institut National des Sciences de l'Univers of the Centre National de la Recherche Scientifique of France, and the University of Hawaii. This publication makes use of data products from the Two Micron All Sky Survey, which is a joint project of the University of Massachusetts and the Infrared Processing and Analysis Center/California Institute of Technology, funded by the National Aeronautics and Space Administration and the National Science Foundation. This work is based in part on data obtained as part of the UKIRT Infrared Deep Sky Survey (found on the UKIRT site). This research has made use of the VizieR catalogue access tool and the Aladin plot tool at CDS, Strasbourg, France. We thank Sebastien Foucaud for providing a version of the SWarp software that includes the sigma-clipping rejection method. The authors acknowledge useful discussions with Simon Angel, Sibilla Perina, Ryan Quadri, Alvio Renzini, Mirko Simunovic, and Jin-Cheng Yu.

Facilities: CFHT (WIRCam, MegaCam) 


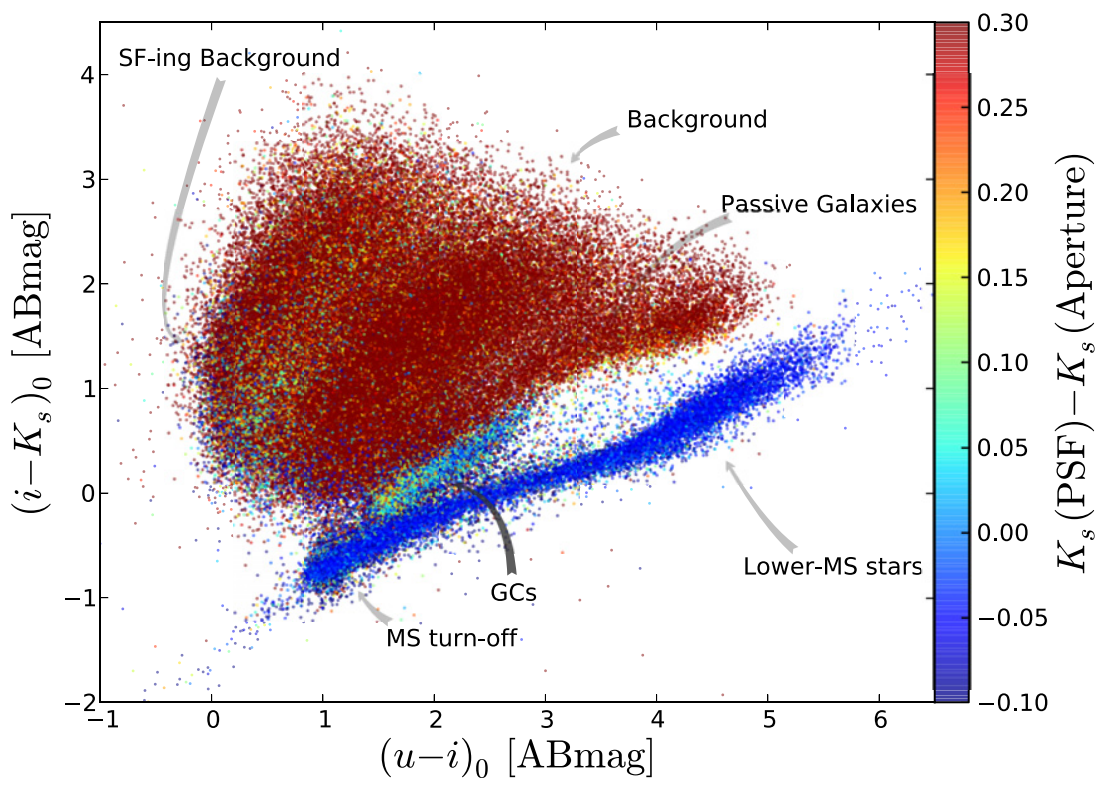

Figure 17. Illustration of the $K_{s}$-band morphology variations across the $u i K_{s}$ diagram. As a measurement of compactness, this figure uses the difference between the $K_{s}$-band PSF magnitude (resulting from the combined used of PSFex and SExtractor) and a magnitude obtained within an aperture with $3^{\prime \prime}$ diameter.

(A color version of this figure is available in the online journal.)

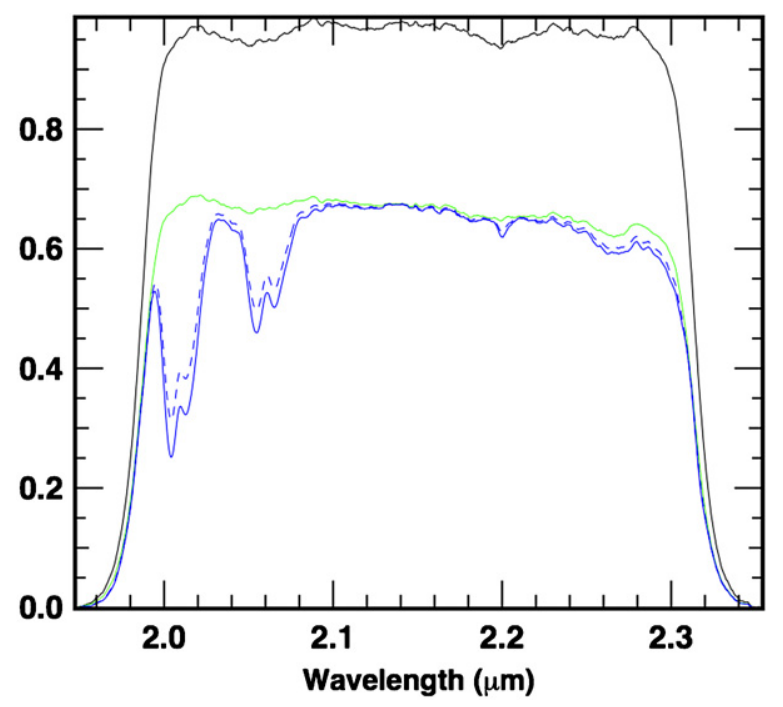

Figure 18. Components of the WIRCam $K_{s}$ transmission used to compute synthetic photometry. The top curve shows the filter transmission. The efficiency of the optics reduces the global efficiency to values between $60 \%$ and $70 \%$ (green curve). The additional effect of telluric features is shown for air masses of 1 (dashed purple curve) and 1.5 (solid purple curve).

(A color version of this figure is available in the online journal.)

\section{APPENDIX A}

\section{WIRCam $K_{s}$ PHOTOMETRY: ADDITIONAL INFORMATION AB MAGNITUDES VERSUS VEGA-BASED MAGNITUDES}

The constant difference between Vega-based magnitudes and $\mathrm{AB}$ magnitudes for the WIRCam $K_{s}$ observations can be obtained from synthetic photometry. The CFHT WIRCam Web pages ${ }^{33}$ warn that various authors have used conversion constants that differ by up to $0.2 \mathrm{mag}$. How the various values

\footnotetext{
33 See http://www.cfht.hawaii.edu/Instruments/Imaging/WIRCam/ dietWIRCam.html.
}

Table 4

Synthetic AB Magnitudes of Vega

\begin{tabular}{lcc}
\hline \hline & alpha.lyr.stis.003 & VegaLCB \\
\hline Filter only & 1.821 & 1.828 \\
Filter+optics & 1.819 & 1.826 \\
Filter+optics+atm. & 1.827 & 1.834 \\
\hline
\end{tabular}

Notes. For various transmission curves and Vega models. An air mass of 1.5 is assumed in the last row. The recommended value is highlighted.

have been obtained, however, is not documented systematically and is rarely indicated in journal publications. We detail our own derivation below.

Figure 18 shows the components of the WIRCam $K_{s}$ transmission curve. The data for the instrument components were obtained from the WIRCam throughput Web pages (files cfh8302.dat for the filter, and WIRCamOpticsResponseCurve.xls for the optics). The optics transmission curve includes all optics except the telescope mirror and tip-tilt plate. We thus implicitly assume these two components have a flat response. The atmospheric transmission for Mauna Kea was obtained from the website of Gemini Observatory. It is based on the ATRAN code (Lord, S. D. 1992, NASA Technical Memorandum 103957) and assumes a water vapor column of $1 \mathrm{~mm}$.

Table 4 provides the conversion constants obtained for WIRCam $K_{s}$ under various assumptions. Three models for Vega were used; alpha.lyr.stis.003 and alpha.lyr.stis.005 are two versions of the standard Vega spectrum distributed by the Space Telescope Science Institute (Bohlin 2007 and the CALSPEC Web page on www.stsci.edu). They differ only shortward of $0.53 \mu \mathrm{m}$, which affects the NGVS $u^{*}$ and $g$ bands but not WIRCam $K_{s}$. VegaLCB is the reference spectrum distributed with the population synthesis code PEGASE (Fioc \& Rocca-Volmerange 1997). The differences from the HST spectra are (1) a globally lower flux level $(0.7 \%)$, (2) a lower spectral resolution (which leads to small differences 
Table 5

Extinction Coefficients for NGVS + NGVS-IR Filters

\begin{tabular}{lcllllll}
\hline \hline Filter Attributes & & \multicolumn{1}{c}{$u^{*}$} & \multicolumn{1}{c}{$g$} & \multicolumn{1}{c}{$r$} & \multicolumn{1}{c}{$i$} & \multicolumn{1}{c}{$K_{s}$} \\
\hline Vega & $A(\lambda) / A(V)$ & 1.490 & 1.190 & 0.874 & 0.674 & 0.498 & 0.118 \\
& Effective $\lambda(\mu \mathrm{m})$ & 0.3895 & 0.4803 & 0.6212 & 0.7493 & 0.8849 & 2.144 \\
Sun & $A(\lambda) / A(V)$ & 1.492 & 1.160 & 0.868 & 0.668 & 0.498 & 0.120 \\
& Effective $\lambda(\mu \mathrm{m})$ & 0.3876 & 0.4906 & 0.6252 & 0.7532 & 0.885 & 2.144 \\
Red star & $A(\lambda) / A(V)$ & 1.462 & 1.120 & 0.858 & 0.654 & 0.492 & 0.120 \\
& Effective $\lambda(\mu \mathrm{m})$ & 0.3967 & 0.5038 & 0.6309 & 0.7620 & 0.8904 & 2.146 \\
& & & & & & &
\end{tabular}

of the integrals over hydrogen features), and (3) local differences in the shape of the spectrum, such as between 2.3 and $2.5 \mu \mathrm{m}$ or around $1.5 \mu \mathrm{m}$. The conversion constant we recommend is

$$
K_{s}(\mathrm{AB})-K_{s}(\mathrm{Vega})=1.827 \mathrm{mag} .
$$

This value is essentially identical to the value obtained by Stéphane Arnouts at CFHT (1.824 mag).

Users of PEGASE should be reminded that code provides Vega-type magnitudes and colors under the assumption that Vega has a magnitude of 0.03 (and not 0.0) in all filters. Therefore, the WIRCam $K_{s}$ magnitudes produced for synthetic populations by PEGASE in the AB system and in the Vega-based system, using VegaLCB as a reference, differ by 1.804 instead of 1.834. Rather than using Vega, our customized version of PEGASE computes AB synthetic magnitudes directly.

\section{APPENDIX B}

\section{EXTINCTION COEFFICIENTS}

We have derived extinction coefficients by comparing the synthetic colors of stellar model spectra and of reddened versions thereof, using the extinction law of Cardelli et al. (1989) with $R_{V}=3.1$. The stellar models used were representations of Vega, of the Sun, and of a red star (in practice, the solar model seen through 3 mag of $V$-band extinction). Table 5 lists the results.

One may compare the values obtained here for CFHT/ MegaCam with those provided for a G2 V star on the stellar isochrone Web site of Padova Observatory, ${ }^{34}$ where the same extinction law is assumed. Their values of $A(\lambda) / A(V)$ for Megacam $u^{*}$ griz are $1.466,1.167,0.860,0.656$, and 0.500 . No comparison value is available through that interface for WIRCam $K_{s}$.

Note that the foreground extinction toward Virgo is smaller, $A(V)=0.1$. Uncertainties associated with neglecting color terms or using one or the other reference for extinction coefficients will therefore be smaller than $\sim 0.5 \%$.

\section{REFERENCES}

Ames, A. 1930, AnHar, 88, 1

Arimoto, N. 1996, in ASP Conf. Ser. 98, From Stars to Galaxies: The Impact of Stellar Physics on Galaxy Evolution, ed. C. Leitherer, U. Fritze-vonAlvensleben, \& J. Huchra (San Francisco, CA: ASP), 287

Bekki, K., Couch, W. J., Drinkwater, M. J., \& Shioya, Y. 2003, MNRAS, 344, 399

Bertin, E. 2006, in ASP Conf. Ser. 351, Astronomical Data Analysis Software and Systems XV, ed. C. Gabriel, C. Arviset, D. Ponz, \& E. Solano (San Francisco, CA: ASP), 112

${ }^{34}$ See http://stev.oapd.inaf.it/cgi-bin/cmd.
Bertin, E. 2011, in ASP Conf. Ser. 442, Astronomical Data Analysis Software and Systems XX, ed. I. N. Evans, A. Accomazzi, D. J. Mink, \& A. H. Rots (San Francisco, CA: ASP), 435

Bertin, E., \& Arnouts, S. 1996, A\&AS, 117, 393

Bertin, E., Mellier, Y., Radovich, M., et al. 2002, in ASP Conf. Ser. 281, Astronomical Data Analysis Software and Systems XI, ed. D. A. Bohlender, D. Durand, \& T. H. Handley (San Francisco, CA: ASP), 228

Bielby, R., Hudelot, P., McCracken, H. J., et al. 2012, A\&A, 545, A23

Binggeli, B., Sandage, A., \& Tammann, G. A. 1985, AJ, 90, 1681

Blakeslee, J. P. 2012, Ap\&SS, 341, 179

Blakeslee, J. P., Jordán, A., Mei, S., et al. 2009, ApJ, 694, 556

Bohlin, R. C. 2007, in ASP Conf. Ser. 364, The Future of Photometric, Spectrophotometric and Polarimetric Standardization, ed. C. Sterken (San Francisco, CA: ASP), 315

Cardelli, J. A., Clayton, G. C., \& Mathis, J. S. 1989, ApJ, 345, 245

Cohen, J. G., Blakeslee, J. P., \& Ryzhov, A. 1998, ApJ, 496, 808

Côté, P., Blakeslee, J. P., Ferrarese, L., et al. 2004, ApJS, 153, 223

Cutri, R. M., Skrutskie, M. F., van Dyk, S., et al. 2003, yCat, 2246, 0

Daddi, E., Cimatti, A., Renzini, A., et al. 2004, ApJ, 617, 746

Dahlen, T., Mobasher, B., Faber, S. M., et al. 2013, ApJ, 775, 93

Dalton, G. B., Caldwell, M., Ward, A. K., et al. 2006, Proc. SPIE, 6269, 30

de Vaucouleurs, G., de Vaucouleurs, A., Corwin, H. G. Jr., (ed.), et al. 1991, in Third Reference Catalogue of Bright Galaxies. Volume I: Explanations and references. Volume II: Data for galaxies between $0^{h}$ and $12^{h}$. Volume III: Data for galaxies between $12^{h}$ and $24^{h}$ (New York: Springer), 2091

Faber, S. M., Willmer, C. N. A., Wolf, C., et al. 2007, ApJ, 665, 265

Fellhauer, M., \& Kroupa, P. 2002, MNRAS, 330, 642

Ferrarese, L., Côté, P., Cuillandre, J.-C., et al. 2012, ApJS, 200, 4

Fioc, M., \& Rocca-Volmerange, B. 1997, A\&A, 326, 950

Forte, J. C., Faifer, F. R., Vega, E. I., et al. 2013, MNRAS, 431, 1405

Gavazzi, G., Boselli, A., Donati, A., Franzetti, P., \& Scodeggio, M. 2003, A\&A, 400, 451

Hanes, D. A., \& Brodie, J. P. 1986, ApJ, 300, 279

Hanes, D. A., Côté, P., Bridges, T. J., et al. 2001, ApJ, 559, 812

Harris, W. E., Whitmore, B. C., Karakla, D., et al. 2006, ApJ, 636, 90

Haşegan, M., Jordán, A., Côté, P., et al. 2005, ApJ, 627, 203

Hempel, M., \& Kissler-Patig, M. 2004, A\&A, 428, 459

Hilker, M. 2011, EAS Publications Series, 48, 219

Jensen, J. B., Tonry, J. L., Barris, B. J., et al. 2003, ApJ, 583, 712

Jones, J. B., Drinkwater, M. J., Jurek, R., et al. 2006, AJ, 131, 312

Jordán, A., Côté, P., West, M. J., \& Marzke, R. O. 2002, ApJL, 576, L113

Jordán, A., Peng, E. W., Blakeslee, J. P., et al. 2009, ApJS, 180, 54

Kim, H.-S., Yoon, S.-J., Sohn, S. T., et al. 2013, ApJ, 763, 40

King, I. R. 1978, ApJ, 222, 1

Kissler-Patig, M., Brodie, J. P., \& Minniti, D. 2002, A\&A, 391, 441

Kotulla, R., Fritze, U., Weilbacher, P., \& Anders, P. 2009, MNRAS, 396, 462

Kurk, J., Cimatti, A., Daddi, E., et al. 2013, A\&A, 549, A63

Lançon, A., \& Mouhcine, M. 2000, in ASP Conf. Ser. 211, Massive Stellar Clusters, ed. A. Lançon \& C. Boily (San Francisco, CA: ASP), 34

Lane, K. P., Almaini, O., Foucaud, S., et al. 2007, MNRAS, 379, L25

Lawrence, A., Warren, S. J., Almaini, O., et al. 2007, MNRAS, 379, 1599

Lawrence, A., Warren, S. J., Almaini, O., et al. 2012, yCat, 2314, 0

Lin, L., Dickinson, M., Jian, H.-Y., et al. 2012, ApJ, 756, 71

Liu, Y., Zhou, X., Ma, J., et al. 2005, AJ, 129, 2628

Ly, C., Malkan, M. A., Kashikawa, N., et al. 2012, ApJ, 757, 63

McCracken, H. J., Capak, P., Salvato, M., et al. 2010, ApJ, 708, 202

McCracken, H. J., Milvang-Jensen, B., Dunlop, J., et al. 2012, A\&A, 544, A156

McDonald, M., Courteau, S., Tully, R. B., \& Roediger, J. 2011, MNRAS, 414, 2055

Mei, S., Blakeslee, J. P., Côté, P., et al. 2007, ApJ, 655, 144

Merson, A. I., Baugh, C. M., Helly, J. C., et al. 2013, MNRAS, 429, 556

Misgeld, I., \& Hilker, M. 2011, MNRAS, 414, 3699

Mouhcine, M., González, R. A., \& Liu, M. C. 2005, MNRAS, 362, 1208 
Park, C., \& Choi, Y.-Y. 2005, ApJL, 635, L29

Park, H. S., Lee, M. G., \& Hwang, H. S. 2012, ApJ, 757, 184

Paudel, S., Duc, P.-A., Côté, P., et al. 2013, ApJ, 767, 133

Peng, E. W., Jordán, A., Blakeslee, J. P., et al. 2009, ApJ, 703, 42

Peng, E. W., Jordán, A., Côté, P., et al. 2008, ApJ, 681, 197

Pessev, P. M., Goudfrooij, P., Puzia, T. H., \& Chandar, R. 2008, MNRAS, 385,1535

Pota, V., Forbes, D. A., Romanowsky, A. J., et al. 2013, MNRAS, 428, 389

Puget, P., Stadler, E., Doyon, R., et al. 2004, Proc. SPIE, 5492, 978

Puzia, T. H., Mobasher, B., \& Goudfrooij, P. 2007, AJ, 134, 1337

Puzia, T. H., Zepf, S. E., Kissler-Patig, M., et al. 2002, A\&A, 391, 453

Raimondo, G., Cantiello, M., Brocato, E., \& Biscardi, I. 2010, MSAIS, 14, 63

Rangel, C., Nandra, K., Laird, E. S., \& Orange, P. 2013, MNRAS, 428, 3089

Reaves, G. 1956, AJ, 61, 69

Reaves, G. 1983, ApJS, 53, 375

Riffel, R., Ruschel-Dutra, D., Pastoriza, M. G., et al. 2011, MNRAS, 410, 2714
Romanowsky, A. J., Strader, J., Brodie, J. P., et al. 2012, ApJ, 748, 29

Schlafly, E. F., \& Finkbeiner, D. P. 2011, ApJ, 737, 103

Schuberth, Y., Richtler, T., Hilker, M., et al. 2012, A\&A, 544, A115

Sick, J., Courteau, S., Cuillandre, J.-C., et al. 2013, arXiv:1303.6290

Skrutskie, M. F., Cutri, R. M., Stiening, R., et al. 2006, AJ, 131, 1163

Strader, J., Romanowsky, A. J., Brodie, J. P., et al. 2011, ApJS, 197, 33

Tamura, N., Sharples, R. M., Arimoto, N., et al. 2006a, MNRAS, 373, 588

Tamura, N., Sharples, R. M., Arimoto, N., et al. 2006b, MNRAS, 373, 601

Taylor, E. N., Hopkins, A. M., Baldry, I. K., et al. 2011, MNRAS, 418, 1587

Tonry, J. L., Ajhar, E. A., \& Luppino, G. A. 1990, AJ, 100, 1416

van Dokkum, P. G. 2001, PASP, 113, 1420

Vogel, H. 1979, Mathematical Biosciences, 44, 179

Webb, J. J., Harris, W. E., \& Sills, A. 2012, ApJL, 759, L39

Worthey, G. 1994, ApJS, 95, 107

Yoon, S.-J., Sohn, S. T., Lee, S.-Y., et al. 2011, ApJ, 743, 149

Yuma, S., Ohta, K., \& Yabe, K. 2012, ApJ, 761, 19

Yuma, S., Ohta, K., Yabe, K., Kajisawa, M., \& Ichikawa, T. 2011, ApJ, 736, 92 